\title{
An Intelligent Semanticification Rules Enabled User- Specific Healthcare Framework Using loT and Deep Learning Techniques
}

Radhika R ( $\nabla$ rr1.techno@gmail.com)

SRMIST: SRM Institute of Science and Technology https://orcid.org/0000-0002-4014-7256

Bhuvaneswari A

Vellore Institute of Technology: VIT University

Kalpana G

Rajalakshmi Institute of Technology

\section{Research Article}

Keywords: Internet of Things, MQTT, SeSem Framework, Semantification rules, Sensor Priority, SS ontology, Deep Learning.

Posted Date: March 16th, 2021

DOI: https://doi.org/10.21203/rs.3.rs-277610/v1

License: (c) (i) This work is licensed under a Creative Commons Attribution 4.0 International License. Read Full License 


\title{
An Intelligent Semanticification Rules Enabled User-Specific Healthcare Framework Using IoT and Deep Learning
}

\author{
${ }^{1}$ Radhika R, ${ }^{2}$ Bhuvaneswari A, ${ }^{3}$ Kalpana G \\ ${ }^{1}$ Assistant Professor, SRM Institute of Science \& Technology,Kattankulathur, Chennai, Tamil Nadu, India \\ ${ }^{2}$ Assistant Professor, Vellore Institute of Technology, Chennai, Tamil Nadu, India \\ ${ }^{3}$ Assistant Professor, Rajalakshmi Institute of Technology, Chennai, Tamil Nadu, India \\ Email : ${ }^{1}$ radhikar2@srmist.edu.in, ${ }^{2}$ bhuvaneswari.a@vit.ac.in, ${ }^{3}$ g.gkalpana@gmail.com
}

\begin{abstract}
Things or device under the Internet of Things, when connected to the internet, becomes a product, offers various applications and services for end-users. Existing methodologies offer sensor-based IoT based health-care services, to the end-users populated with lots of sensed values all at a time, making the health-care system with least robustness and inefficient due to unsystematic preview of patient's health record. In order to address this issue, a novel semantic-based service framework is proposed in this paper which allows the end-user to subscribe for a specific physiological parameter, among all the available sensed data, making the health-care system more efficient. In the SeSem framework, Semantification rules and semantification relationship table are applied to the sensed JavaScript object Notation format (JSON) data, in order to semantically separate the JSON data, into a meaningful format. To list the sensed data according to the significance of health issues, a priority is assigned to the sensors in the semantification relationship table. Hence, semantically separated data can be done along with assigned priority. Sensor-based Sematic (SS) ontology is then applied to the semantically separated data, to transform the sensed data more relevant in terms of particular disease and sensor associated with it. The semantically separated sensed data are then published to the Message Queuing Telemetry Transport (MQTT) interface. Using MQTT subscribe, the end-user along with date and time, requests for a particular service, using a Semantic Similarity mapping algorithm, which compares the entire sensed data to that of requested data and responds with a particular physiological parameter request. To make the health care system deploy services in an intelligent way, deep learning algorithm Feedforward Recurrent Neural Networks are applied, which makes the prediction of sensed data based on the latest update when the end-user subscribes for a certain sensed data without specifying date and time. The proposed methodology is evaluated against IoT performance metrics which had shoed showing better results in terms of service-oriented IoT.

Keywords: Internet of Things; MQTT; SeSem Framework; Semantification rules; Sensor Priority; SS ontology; Deep
\end{abstract} Learning.

\section{Introduction}

Internet of Things is a trending technology contributing several solutions providing various applications and resources, leading to the rapid growth of industrial support in a smart way, by interconnecting devices to the internet. There exisits various smart devices, which generate diverse amount of sensors that are in-built within smart devices like mobile phones, wearable devices, alert systems, vehicles, smart homes and so on. The sensor in the car may indicate the driver about the forthcoming traffic, speed breakers in the road, the type of landscape the car is approaching and so on. This intelligent identification is possible by immeasurable advancements in Radio-Frequency identification, wireless sensor networks, mobile and sensor devices [1]. With the combination of these smart devices and interconnection technologies, IoT offers a wide variety of services to the users namely, E-learning, temperature information, location and automatic switching of lights on or off at smart homes. Depending upon the user's requirements, the type of smart device and the sensors associated with the devices may vary.

In the real-world world scenario, different types of users require different delay latencies. In order to achieve user-defined IoT services, Software Defined Networks publish or subscribe architecture and eXtreme Gradient Boosting (XGBoost) machine learning model [2] was proposed promising various delay-sensitive IoT services in order to improve the QoS in SDN-like publish or subscribe middleware. In most of the service-based IoT systems, 
even though they are user-specific, they can offer only a single kind of service, has a limitation of providing ondemand dynamic user-preferences based services. In [3], the IoT service aggregation method is proposed which considers both functional and non-functional requirements of the users, by semantically computing the sensed data by applying service category based IoT ontologies. From the observed data,the IoT service selection algorithm is chosen which provides varying IoT services according to user's preferences and environment.

In [4], a patient health monitoring system was proposed using various sensors like temperature sensor, bed wet sensor, accelerometer sensor in an AD8232 module enhanced in a microcontroller and MQTT protocol for publishing the health status of the patient to the caretaker and doctor. The system performs early detection of the patient's abnormal health condition, by applying data mining to the gathered knowledge through sensed data. In [5], the author proposed a framework for a semantic finding of services, in swarm intelligence. This framework, allows the user to specify services of their choice. The semantics involved in this framework allows for the accurate specification of services in terms of both input and output. JSON-LD and Hydra allow the specification of services in a user-friendly manner. MQTT based remote health monitoring system was proposed in [6], in which OMNET ++ was used to implement the proposed design, using the INET framework. This work has overcome the issue of decreased MQTT broker performance when the number of publishers and subscribers increase in number. The usage of MQTT protocol in the proposed framework has higher throughput and lower end-end delay when compared to the HTTP protocol. In [7], MQTT protocol bases remote ECG monitoring was proposed, using Raspberry pi as a prototype. The mosquito MQTT client program captures the body vital signs and publishes it to the MQTT broker as per topics. The end-user using MQTT subscribe on a particular topic can be able to view data visualized format, to make the doctor analyze the patient's current health status in an accurate manner.

All of the above discussed IoT services with respect to health scenarios capture the vital signs of patient health through the sensors worn or implanted on the patient. The sensed data will be in a tremendous amount which makes the assessment of the patient's health by the end-users a challenging task. Temporal rough set induced neurofuzzy (TRiNF) mining framework [38], is an existing methodology, which efficiently handles the complexities in the time series based clinical dataset in terms of irregular observations and missing values. TRiNF implements two functionalities namely, Temporal Data Acquisition (TDA) and temporal classification. Though the classifier accuracy of TRiNF is higher when compared to all existing methodologies, still research has been carried out to improve the classifier accuracy in a far better way. Neural Network classifier using Differential Evolution with Global Information and Back Propagation (NN-DEGI-BP) algorithm for clinical datasets [39], is a Computer-Aided Diagnostic (CAD) methodology, which helps in assisting the doctors in decision making for disease identification. But this classifier showed up less classifier accuracy and decreased reliability when the datasets with time-series information are considered.

In order to address this challenging issue, this paper proposes a novel semantic-based service framework, Sensor-based Semantic (SeSem) framework, in which an end-user can subscribe for the intended physiological parameter of interest, for better understanding and monitoring of patient's health status. In the SeSem framework, the populated sensor data are stored in JSON format in Data storage. For providing an accurate semantic description, a set of semantification rules and semantification relationship tables are utilized; making the unorganized sensed data into a meaningful interpretation by semantically separating the sensed data. To prioritize the patient's health record based on a particular syndrome impact, a priority is assigned to the sensors in the semantification rules table, so that the semantic separation can be done based on the priority of the sensors. Sensor-based Semantic (SS) ontology, has also been proposed in SeSem framework, making the semantically separated data in a more meaningful way, separating sensors on based disease, priority, and patient and so on. A semantic similarity matching algorithm called semantic similarity mapper has been proposed. It is used for mapping the subscribed physiological parameter along with date and time from the published semantically separated based sensor data. Few subscribers might not specify date and time, while subscribing for a particular sensor data, which might lead to the same issue of populating tremendous data of particular physiological parameter, with various date and time. To overcome this issue, a deep neural network, with recursive type is applied to the matching subscribed data, which helps in providing the Last updated date and time service, to the end-users, thus making our health-care system an intelligent system. 
The research paper is organized follows: In section 2, the previous work related to IoT services, IoT interoperability, IoT ontologies and Machine Learning approach for IoT based services and applications are discussed. Section 3 discusses IoT interoperability issues in various applications and services and the types of IoT interoperability. Section 4 summarizes the importance of IoT gateway and middleware. Section 5 discusses IoT protocols and the different protocols used in IoT layers. In section 6, various IoT protocol interoperability solutions proposed in previous works are discussed. Section 7 discusses the problem and its proposed solution. Section 8 discusses result analysis and Section 9concludes the paper.

\section{Related works}

This section discusses the works related to IoT based services, uses various ontologies and techniques offering a variety of user-specific, industry-specific and generalized IoT services. It also discusses papers speaking about semantic interoperability, a great limitation in IoT, and the efforts to eradicate interoperability. Discussion about various intelligent algorithms applied for various IoT based applications is also given in this section.

\subsection{IoT as service}

Nachabe et al [8] proposed an OntoSmart semantic multi-agent architecture, offers automatic service discovery that is fully interoperable. The sensors and actuators used in the distributed architecture are independently leading to a smart interoperable IoT system. Simone Cirani et al [9] have proposed a large scale peer-peer IoT architecture, employs local and resource directory, for automatic service detection without human intercession. Meesun Kim et al [10] propose process-aware IoT services, in which AURDINO community computing environment and its technical services are used in order to study the viability of process-aware Internet of Things. Luca Davoli et al [11] proposed an Internet Protocol version 6 (IPv6) Routing Protocol for Low-power Lossy Networks (RPL) based IoT services, named as RPL based Resource and service discovery algorithm (DiRPL), uses CoAP protocol for sending request and the result will be published in a local directory. DiRPL supports very minimal human intervention and selfconfigurable with the help of the Service Directory (SD) and Resource Directory (RD). Ching-Hu Lu et al [12] proposed three modules namely Market-And-Discover (MnD), Market-And-Recommend (MnR) and Market-AndInteract (MnI) for ensuring a cross-field flexible, reusable and reconfigurable user-centered IoT services. MnD collects data from cross-fields based on the user's interest. The user can manage their personal preferences in order to ensure privacy. MnRhelps the user to get only matching advertisements as per data collected by $\mathrm{MnD}$ so that unwanted advertising can be avoided. MnI provides the user with reconfigurable cross-field services, where the communication becomes bidirectional. George Kousiouris et al [13] proposed the AffectUs framework, supporting micro-service based system architecture, which is implementing the integration of various significant elements in the supply chain management namely, semantics, Artificial Intelligent, integration technologies and IoT management platforms in order to uplift the level of on-demand awareness in the supply chain and its related dependencies.

\subsection{Sensor data and semantification}

Xiang Zhang et al[14] have proposed a multi-modality sensor-data classification framework, which is a combination of selective-attention mechanism, deep reinforcement learning, and WAS-LSTM classification. Their framework, overcomes the domain-specific methodologies thus improving the adaptability of classification algorithms in various applications.Jean Paul Bambanza [15], developed the iSEE system, a semantic sensor selection system, consisting of four layers namely, the application layer, User layer, ontology layer and systems layer. This system enables the enduser to select IoT devices for a specific health-based scenario using Smart HealthCare (SHO) prototype along with Disease Ontology (DO). The iSEE system, allows the end-user to use semantic health care ontology, from where desired IoT devices can be selected and a detailed description of the selected device can also be obtained. Rustem Dautov et al [16] proposed distributed hierarchical data fusion architecture, which combines the data collected from several sources at each level of IoT layers, with minimum delay and immediate response.

The proposed architecture has made advancement in the health care domain, by overcoming the time constraint issue and device heterogeneity. GergelyMarcell Honti et al [18] have reviewed the semantic sensor technologies on the internet of things. This review talks about the sensor data that can be made semantically separable, by collecting semantic metadata and applying different sensor ontologies, to transform the sensed data into a 
meaningful format.Abdullah Alamri [19] proposed semantically interoperable middleware, for IoT data and EHR data. This middleware enables interoperability, integration, information hunt and recovery, and automatic conclusion. Their middleware helps in configuring IoT in HER, by collecting actionable data, which will, in turn, improvise the patient care by appropriate treatment and interpolations with respect to the actionable data collected. Argyro Mavrogiorgou et al [20] proposed a data collection and representation mechanism of four stages, in which at the first stage the data from all heterogeneous health-based sensor data are collected. Next, the cleaning of the collected dataset occurs, which drops missing and noisy values in the dataset. This cleaned data is evaluated against the data quality measurements, which were collected from various devices and data from a device with only high-quality data standards are retained. Finally, the cleaned and high-quality data are transformed into a common format, thus providing interoperability. Ajigboye et al [26] proposed the ontology-driven se-HIS with the aim of promoting semantic interoperability in the healthcare systems. A framework named SIOP has been proposed which is ontologybased and inherited from the previous standards has been used. The ontology is driven from heavyweight IEEE 21450 family standards. SIOP framework is vigorous as it can handle both sensors and actuators. Ayaskanta Mishra et al [27] have proposed an MQTT based remote ECG monitoring system. The raspberry pi is used for prototyping their proposed design. MQTT client collects data from the AD8232 ECG sensor module and publishes the data to the MQTT broker in the cloud. The end-user can subscribe to the particular ECG related parameter and visualize it as a graphical format. Jane et al., [38] have proposed temporal rough set induced neuro-fuzzy (TRiNF) mining framework, for handling the complex clinical datasets. This framework implements two functionalities namely 1) Temporal Data Acquisition (TDA) used for missing value assertion and temporal pattern extraction and 2) Temporal classifier, for classifying attributes based on Temporal pattern.

\subsection{IoT and deep learning}

Mohan Kumaret al.,[28]talked about the intelligent way of responding to the desired request imposed by the end-user by applying machine learning paradigms as a backend. This paper has discussed the possibilities of combining cloud computing and machine learning paradigms into distributed computing based on the IoT framework. By doing so, the desired information of interest can be extracted from the huge data set and by applying the intelligence paradigm; data can be acquired based on priority. Abdul Salam et al [30] have proposed a QoS algorithm that provides three classes of services based on the type and severity of the sensors. From the experiments, it is found that guaranteed services for critical sensor nodes, real-time services for less critical nodes and best efforts services for other sensor nodes. Ademola Philip Abidoye et al [31] have proposed a differentiated service scheme based on priority scheduling and data compression. This scheme is implemented in the second tier of remote health care architecture. This prioritization has decreased the transmission delay in transmitting vital signs of the physiological parameter of the patient to the end-user. This approach has also reduced bandwidth utilization. Sapna Gambhir et al [32] have used a new class of intelligent networks named Wireless Body Area Network (WBAN), in order to sense and group the sensed data into different classes. A central node observes for any changes in the observed data, and if it happens it assigns it a higher priority and sends an alert message to the medical server. A node with high priority is assigned more network bandwidth than other classes of nodes. Thus making the end-user like doctors and nurses take quick actions based upon the sensed values related to the patient's physiological parameter.

O. S. Albahri et al [34] reviewed the three architecture used in IoT for collecting sensed data and sending it to tier-2, consisting of the gateway of local area networks. The sensed data is then sent to the medical server in tier- 3 using IoT protocols and makes an alert. This paper has also made a survey on priority based sensor data, where the sensed data with abnormal signs are assigned a higher priority than others, resulting in higher network bandwidth allocation for higher priority sensor nodes. Jie Tang et al [36] have discussed the different IoT devices being used in different fields. This research paper has notified how and why various deep learning algorithms are applied in order to make unstructured multimedia data into a structured and intelligent manner.

\section{IoT interoperability in HealthCare Systems}

In [37], it is clear that the inability of a smart device to communicate with another smart device, in which both the devices have different IoT infrastructure, protocols, APIs, and data formats is termed as IoT interoperability. This is 
the most challenging scenario in the present IoT technology, as many industries and business corporate depend on IoT applications and services. Hence due to IoT interoperability among smart devices, cross-platform applications and services cannot be deployed in IoT expands IoT to the largest scale. Interoperability can be classified as Device interoperability, Network interoperability, Semantic interoperability, Syntactic interoperability and platform interoperability [37].

Device interoperability: The ability of different types of devices, namely low-end devices having insufficient resources, processing power, and communication techniques and high-end devices with enough resources and power, to communicate with each other using varied communication protocols is termed as Device interoperability.[37]

Network Interoperability: The ability of heterogeneous devices to communicate with each other through different networks like Low powered wire-less network, IEEE 802 standards, etc., for an end-end communication is termed as Network Interoperability.[37]

Syntactical Interoperability: The sender and receiver's message will have a different data structure. Hence in order to make them understandable, a channel is provided where the messages are serialized pertaining to the certain schema. The sender before sending the message will encode using syntactic rules and the receiver will have some different syntactic for decoding the message. The Incompatibility of between the sender's encoding rules and receiver's decoding rules leads to the problem of Syntactical interoperability [37].

Semantic interoperability: IoT has various applications and services that have different data formats (XML, JSON, and CSV). Due to this issue, IoT systems might interpret the meaning of data according to their domain and environment. Providing this understanding of information and data in a meaningful way among different dissimilar sources is termed as Semantic interoperability [37].

Platform interoperability: There are plenty of Operating systems, programming languages and data structures, and access mechanisms. Hence, an IoT system might contain smart devices with the above-mentioned differentiations. The ability to pass messages between smart devices with diverse platforms leads to Platform interoperability [39].

\section{Sensor Interoperability}

Sensors and actuators are two important concepts of IoT. The sensor senses the changes in the environment and alerts the concerned end-users through IoT systems. This ability of the sensor to sense and capture the environmental conditions led to the usage of sensors in various fields namely traffic systems, health care, educational institutions, weather forecasting, agriculture, and soil forecasting. Each of these domains has various sensors with their own specifications. Consider Health care systems as a sample scenario. The health care monitoring systems have various sensors like brain wave sensors, Electrocardiogram sensors, blood pressure sensors, skin sensors, electromyography sensors, blood saturation sensors, blood glucose, and monitor sensor. Each sensor will be having its own data standards and data type. Hence the captured data will not be in a meaningful format, leading to sensor interoperability at the semantic level, making the sensor-based systems a failure system. This sensor-based semantic interoperability issue can be solved by constructing ontology, which makes the sensed raw data into a meaningful format, providing interoperability among any kind of sensors under consideration.

\section{Health care systems}

Health care systems in IoT play an important role, in the field of medicine as it makes it possible for effective health monitoring remotely with the usage of different wearable and non-wearable sensors. With these sensors, automated alerts, and remote monitoring of patients by doctors and nurses makes healthcare systems faster and effective in terms of economy. A typical healthcare system consists of different types of sensors worn by different patients. These sensors sense the changes in the patient's health-related signs and send these changes to the database with the updated units. If the signs like heart rate, temperature, blood pressure, sugar level exceed the threshold, an automatic alert will be sent both to the doctor and the patient's caretaker. The doctor, nurse or caretaker can view the current health condition of the patient, by remotely accessing the smart health system and the doctor can give feedback on the status of the patient's health issue now and then. 
The sensor information collected may vary according to different sensors, leading to different data labels. A chest sensor will have heart rate, speed of heart rate while relaxing, speed of heart rate while jumping, jogging, standing and so on. The sensor at the ankle measures the flexibility in terms of vigorous walking, strenuous jobs, and strain rate based on every action. The data sensed by the sensors will not have a meaningful interpretation, in terms of parameters associated with a particular sensor with respect to a particular disease. This sensor-based heterogeneity issue can be addressed by semantically making the data format in an understandable format, providing accurate and robust health care systems. Basically, most of the health care systems, with the help of IoT protocols update the sensed values to the server, in anyJSON or XML format. The end-user might be requiring a particular record of the patient, but the sensed data might contain all the records of the patient based on the number of sensors worn by the user. This leads to high computational costs and affects the smartness of the health care systems. The semantics are used in various research work [40], [41], [42] to identify the information sensitivity

\section{Proposed Framework}

In order to address the above-mentioned issues, our proposed semantics-based services, SeSem frameworkutilizes MQTT publish/subscribe protocol, in which the sensed data along with patient details are published as individual topics to the MQTTinterface. The sensed data will contain all the data sensed by all types of health-related sensors, leading to interpretation issue, in terms of which data belongs to which senor. In order to address this heterogeneity problem, our proposed SeSem framework applies semantification rules and semantification relationship table which semantically separates the sensed data into a meaningful format. To prioritize the sensors based on disease criticality, a priority is assigned to every sensor used in the health care systems. This priority is set through the semantification relationship table, as the semantically separated data can be separated based on sensor priority. The semantically separated data is then applied to sensor-Based Semantic (SS) ontology, which maps the sensed data with respect to a patient's disease and his worn sensor. The end-user on the other hand, using the MQTT subscribe will request for particular data along with the patient name, patient id, date and time. In order to fetch only the requested sensor value, our work implements a semantic similarity mapping algorithm, semantic similarity mapper which performs the calculation of semantic similarity in terms attributes of the sensed data between the published data and subscribed data. After applying the similarity mapping algorithm, the end user's subscribed data will be sent, resolving heterogeneous data issue and high speed and less computational cost based IoT healthcare systems. To make the proposed SeSem framework to deploy services intelligently, a robust scenario is considered where the end-user might subscribe for a particular sensor data, without specifying date and time. In such cases, the mapping algorithm will populate millions of data with the requested physiological parameter, making the system unstable. Hence our framework applies deep recursive feed-forward neural algorithms to the matching data and predicts only the lastly updated sensor information to the subscribed end-users. Using RNN, doctors might analyze a particular sensed valve in terms of other sensed physiological parameters based on minimum,-maximum threshold and provide necessary treatments to the patient. Thus by applying Deep feedforward Recurrent Neural Network, our proposed SeSem framework intelligently deploys services thereby increasing the efficiency and robustness of the remote health care systems.

\section{SeSem (Sensor-based Semantification) Framework}

Health care technologies rely on wearable sensors worn by patients, responsible for identifying and detecting changes in the body condition of the patient. The wearable device can be a single device, accommodating multiple sensors like the blood oxygen sensor, heart rate sensor, temperature sensor or the patient can wear, an individual wearable device for each sensor. The device with the sensor for sensing a particular physiological parameter can be either wearable or implanted. A single wearable device can sense blood pressure level, oxygen level, body temperature and glucose level with the presence of blood oxygen sensor, heart rate sensor and temperature at the same kit. The sensors can sense and update the changes to a database or server with the help of connecting technologies like Bluetooth, zig-bee, and z-wave. With the help of these communicating technologies, IoT is able to intelligently detect and react to the changes accordingly to the environment, remotely reducing the manpower time and computational cost. But due to the usage of heterogeneous sensors and actuators, fetched data becomes complex. 


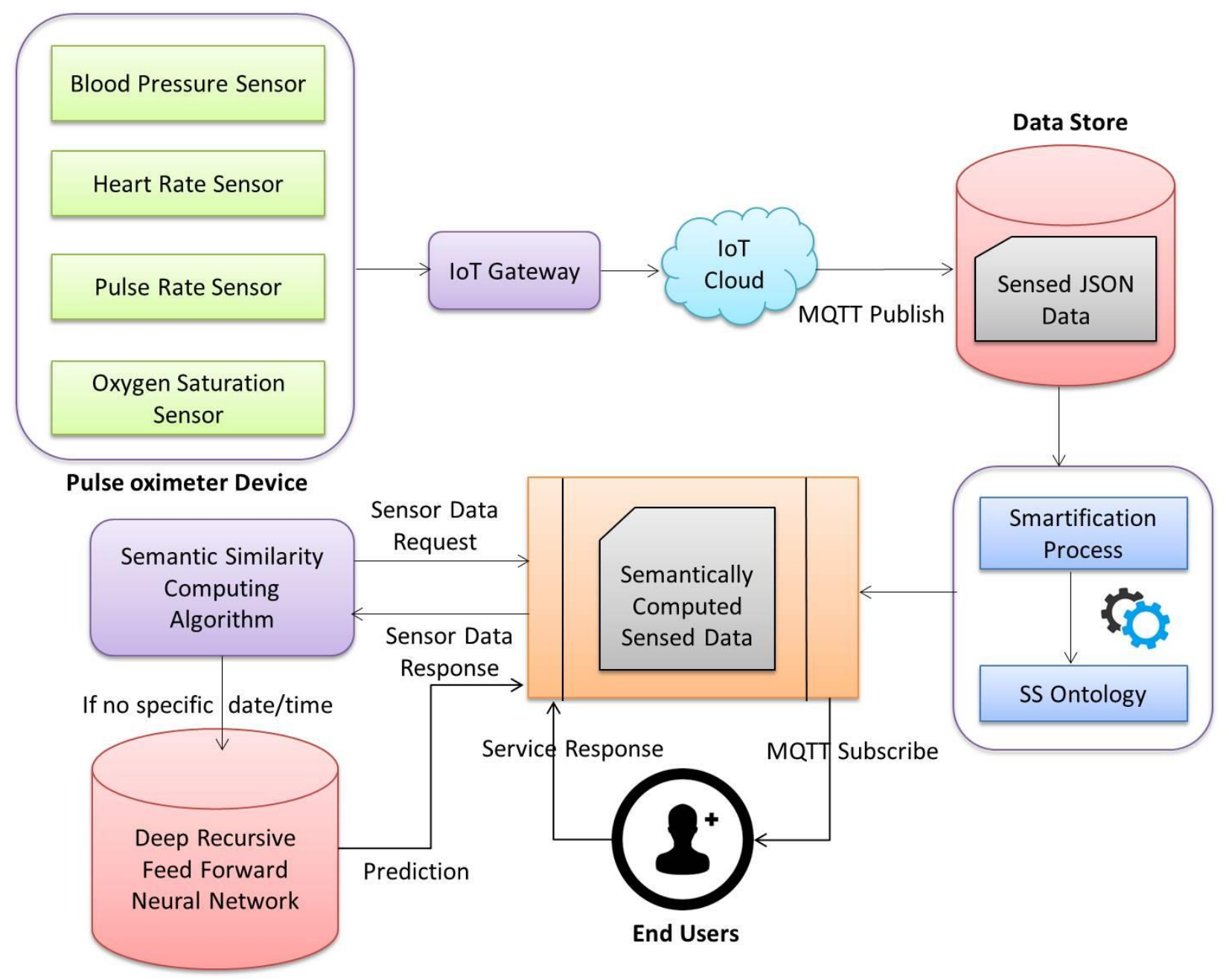

Figure 1. Proposed SeSem Health care system

Figure 1 depicts our proposed healthcare system. In our proposed work, three sensors are considered namely 1, 2, 3 in the healthcare system. All these sensors through IoT gateway populate the sensed data to a database. These data are then fed to our SeSem framework, which performs the semantification process and SS ontology mapping in order to semantically separate the fetched sensor data in a meaningful manner. The resultant semantically separated prioritybased sensed data are published to the MQTT interface. The end-user then subscribes for a particular physiological parameter along with a date and time. This subscribed data is fed to the semantical similarity mapping algorithm,semantic similarity mapper which maps for the particular the subscribed parameters. The mapped values are updated to the end-users based on the priority of the sensors. If data and time are not specified, the mapped values are fed to the deep recursive feed-forward neural network, which predicts and services the end-user with the currently updated sensor value.

A mechanoreceptor is a wearable/ inserted into body sensor, which blood pressure of hypertension cases. ECG sensor is used to detect ECG and cardiac output rate. The thermistor is used to inspect vascular diseases, which will measure blood pressure rate, peripheral perfusion, anerium sac pressure, and body temperature. The ECG sensor can also be used for monitoring patients after operation, which measures heart rate, blood pressure, ECG, oxygen inundation, and body temperature. Each sensor has different data types with different units namely, the temperature can be in Fahrenheit or Celsius, Blood pressure will be in mm units, and heart rate will be BPM (Beats per Minute) and so on. Every sensor differs from its physiological parameters and unit measurements. Hence any system that utilizes IoT as remote monitoring systems must have unique device id, having any type of sensors and the network address of the device registered in a network. The sensors should be responsible for sensing the changes in the 
environment and updating it to a database or server. These sensed data must be passed or communicated to other enduser devices, with the help of IoT communications standards like 802.15.4, Near Field Communication (NFC and Ultra-Wide Bandwidth (UWB). With the help of IoT hardware like Aurdino, Raspberry Pi and software functionalities offering simulator like Contiki, Tiny OS the communication between devices and data interpretation in terms of sensed values with respect to a particular also happens in an IoT network. With these functionalities IoT can extend its services remotely to the end-users or makes the communication between the device to device in an efficient and timeconsuming manner.

Almost every health care system updates all the sensor values to a cloud or data store, from where the sensed physiological changes are notified to the patient, patient caretaker, and doctor responsible for the patient treatment. These sensed values will be complex that, the end-user might suffer from misperceptions due to excess amount of sensed values and the prediction of patient's health, which requires some time as all the labels under particular sensors are updated to the base. In order to address the sensor-based semantification problem and heterogeneous sensed values, our work proposes a framework called Sensor-based Semantification (SeSem) framework, where MQTT protocol is used for sensor devices and end-user communication. Here the end-user can specify the patient's id and name along with the disease name, a particular physiological parameter and date and time (optional). Hence the end-user can obtain the current state of a particular physiological parameter under consideration, avoiding unnecessary parameters, thereby improving the computational process fast and accurate prescription or prediction by doctors and nurses. The energy in fetching the required label under consideration alone, when compared to dumping whatever values fetched, provides our approach much more optimized when compared to the existing health care systems.

In the SeSem framework, the sensed values will be in JSON. It is a subset of JavaScript and when compared to XML it is very easy for machines for parsing and generating. The JSON can have two formats:

$>$ Collection of name-value pairs.

$>$ Ordered list of values

These JSON objects can be stored as file, if we just want to store and less frequent object retrievals. We can also save json objects in database format. Let us assume our healthcare system has pulse oximator sensor device, which records heart rate, pulse rate, respiratory rate and blood oxygen level [43] [44]. These sensors will update its sensed values as the following sample format.

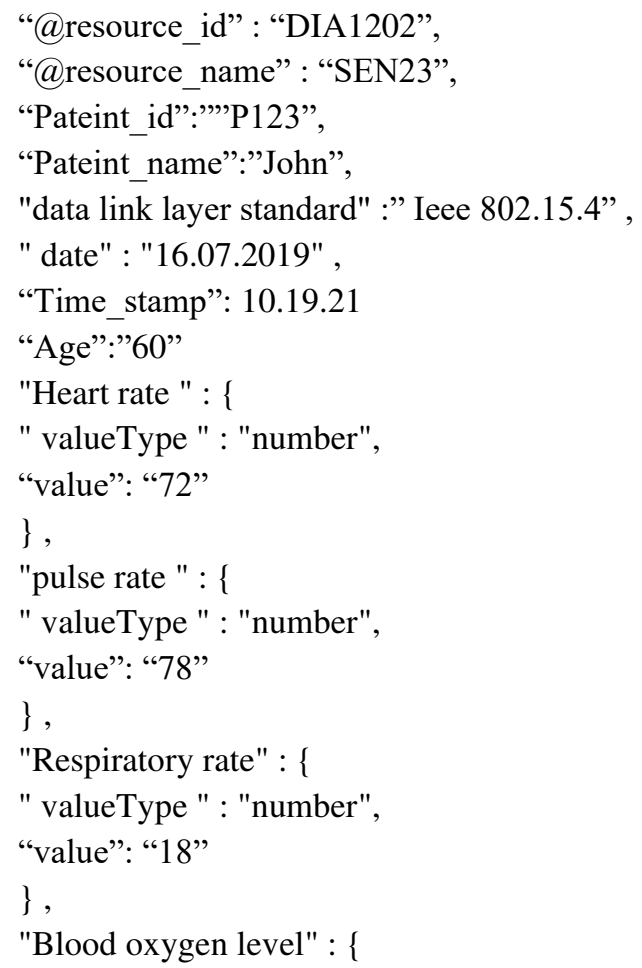


" valueType " : "number",

"value": "88"

\} .

The mentioned JSON objects are samples, wherein at the real scenario, the JSON object will be populated with thousands of observations, for a particular interval of time. When the same is passed to the end-users like patient care or patients apart from doctors and nurses, there won't be a clear understanding of which sensors these values are being updated. In order to provide a meaningful interpretation, the SeSem framework utilizes the semantification rules and relationship table, which helps in semantically separating data with respect to sensors and patients in a clear and meaningful manner. In the semantification relationship table, we are assigning priorities to every sensor connected to the network, which helps in priority based semantically separated data derivation. To interpret in a more meaningful way of sensed data, our framework constructs a Sensor-based Semantic (SS) ontology. This ontology helps in mapping the sensed data to a particular disease, patient and other useful parameters. The MQTT published sensor data is now semantically separated into a meaningful format and hence it is open to the subscriber's request. The health care system end-user, now subscribes for a service, by specifying patient id, disease name, physiological parameter along with/without date and time. The proposed semantic similarity mapping algorithm is implemented,semantic similarity mapper which maps the subscribed parameters to the published sensor data and offers the service of interest to the end-users. If the end-user didn't address any specific date and time, semantic similarity mapped data will contain millions of data of particular requested physiological parameters and hence, a deep recursive feed-forward neural network algorithm is applied to the mapped parameters, which predicts and services only the currently updated sensor value. Thus our proposed SeSem framework is robust and efficient with its additional quality of deploying services intelligibly.

\subsection{Sensor-Based Semantification}

In order to make the data fetched from different sensors to a meaningful interpretation, we need to apply semantification rules to make the IoT system robust in terms of message communication between two devices, which are heterogeneous in nature. This semantification rule results in a meaningful separation of sensed data with respect to sensors and diseases. This semantification process involves two processes: 1) source mapping 2) sensor explanation. The source mapping consists of sensor information like sensor number, patient details, sensed values and units in a generalized manner. The sensor explanation consists of concrete sensor-based information from a particular data source.It consists of device id and rudimentary information about sensors. Figure 2 represents sematificatio rules diagramatically. In semantification rules diagram, source information consists of details of various sensors used in the application and the sensor mapping details about the sensor details like sensor number, patient id, values, and units. Here the sensor number is a unique Id for every sensor being used in the health application. The patient Id corresponds to a particular patient using the health care application, values represent the sensed values and units represents the measurement units for every sensed value. Sensor properties contain the information for a particular sensor. Sensor id, source id, type various sensed values and its units. It talks about the particular sensor type, its id, and parameters involved with respect to a particular sensor. Thus from the semantification process, the data is separated meaningfully in terms of where the sensor values are being populated. Algorithm 1 depicts the generation of semantification rues, which is a basic building block for semantification process. The semantifcation rule generation algorithm takes source information $\mathrm{s}_{\mathrm{i}}$ and source mapping $\mathrm{s}_{\mathrm{m}}$ as inputs and gets the source node $\mathrm{s}_{\mathrm{n}}$ and its corresponding sensor list, $\mathrm{s}_{l}$. From $\mathrm{s}_{\mathrm{n}}$ and $\mathrm{s}_{l}$, we can get sensor mapping and its associated sensor properties as sematification rules. 


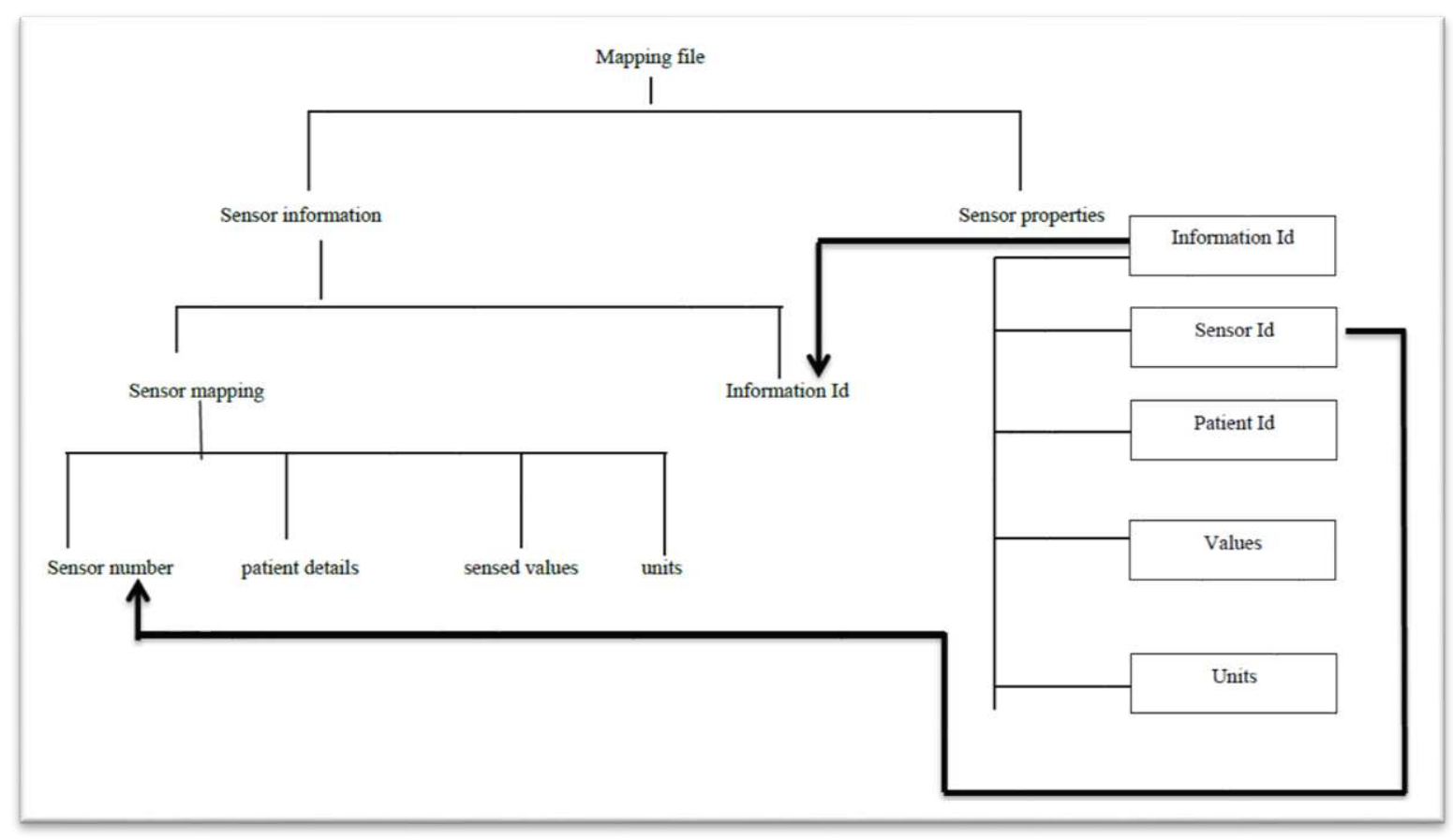

Figure 2. Sematification rules

After the semantification process, we need to use ontologies, which provide a semantic explanation of sensed data as well as providing the reliability of the fetched sensor data. Our proposed framework utilizes Sensor-Based Semantic (SS) Ontology, which is applied to the semanti fied data, for providing more meaningful interpretation regarding, the disease type, sensor used for a particular disease type, sensor information and patient details. By doing so, the disorganized nature of the original sensed object will be resolved, resulting in a meaningful data interpretation. The resulting sensed object will have the name of the sensor, the disease for which the sensor is applied with other sensed object details, in a clear and meaningful manner. From the semantification rules and SS ontology, a semantification relationship table can be derived, which gives the relationship between semantically separated sensed data and SS ontology. Table-I details about the semantification relationship table. In the semantification table, Priority plays an important role, as it assigns priority to the sensors based upon the type of disease. In our pulseoximetry device the highest priority is assigned to blood pressure sensors, followed by heart rate sensors, pulse rate sensors and oxygen saturation leven sensors. This assignment of priority plays a vital role in offering intelligent and fast services, assisiting in right direction to both patient's caretaker and doctors.

Table-I Semantification relationship table

\begin{tabular}{|c|c|c|}
\hline Semantification property & SS ontology property & Description \\
\hline Information number & SS: Sensor Id & The serial number of sensors \\
\hline Patient details & SS: Patient Id & Details of registered patients \\
\hline Sensed values & SS: value & $\begin{array}{c}\text { The value of the measured } \\
\text { physiological parameter }\end{array}$ \\
\hline Units & SS: units & Units of measured values \\
\hline Disease & SS: Disease name & Disease for which sensors are fitted \\
\hline Priority & SS: Priority & $\begin{array}{c}\text { Assigns sensor a priority based on } \\
\text { disease category }\end{array}$ \\
\hline
\end{tabular}

Thus, the semantification process fills the gap between the fetched sensor data and ontology instances, by providing, mapping file and collections of the relationship between, the sensed data and instances of the ontology, which is depicted in algorithm 1 . 


\begin{tabular}{|l|}
\hline Algorithm 1: Semantification rule generation \\
\hline Input: source information $\mathrm{S}_{\mathrm{i}}$, source mapping $\mathrm{Sm}$; \\
Output Semantification rule generation $\mathrm{S}_{\mathbf{R}}$; \\
Step 1: parse $\mathrm{s}_{\mathrm{i}}$ to obtain source node $\mathrm{s}_{\mathrm{n}}$ information; \\
Step 2: get sensorList $\mathrm{ss}_{l}$ from $\mathrm{s}_{\mathrm{m}}$ information; \\
Step 3: for each source node in $\mathrm{s}_{\mathrm{n}}$ \\
Step 4:for each sensor List in sensor Lists \\
Step 5.1: generate sensor mapping \\
$\quad$ Step 5.1.2:generate sensor properties; \\
$\quad$ End For \\
Step 6:End For \\
Step 7: Obtain Semantification rules $\mathrm{S}_{\mathrm{R}}$ consisting of sensor mapping and its associated sensor properties. \\
\end{tabular}

In algorithm 2, the semantification rules are parsed in order to obtain the source, from where the sensor details are coming from. From the source information, the list of sensors is obtained. From which the source mapping consists of sensor information like sensor number, patient details sensed values and units in a generalized manner. The sensor explanation consists of concrete sensor-based information from a particular data source. It consists of device id and rudimentary information about sensors. From the datastore containing sensed values, based on connected sensors to the source are populated. From the result collection, properties associated with individual sensors are obtained as ${ }^{\text {sensor }}$ and I ${ }^{\text {senanno }}$ are generated. From the I ${ }^{\text {senaano }}$, individual sensor explanations take place with respect to sensor annotation senAnno. Using the semantification table list $\mathrm{L}$, a mapping is performed between $\mathrm{I}^{\text {sensor }}$ and $\mathrm{I}^{\text {sensorAnno }}$, from which $I^{\text {sensoroutput }}$ with respect to ${ }^{\text {sid }},{ }^{\text {Pid }}, I^{\text {type }}, I^{\text {value }}, I^{\text {units, }}$ and $I^{\text {sensor }}$ with ${ }^{\text {sensoroutput }}$ can be obtained.

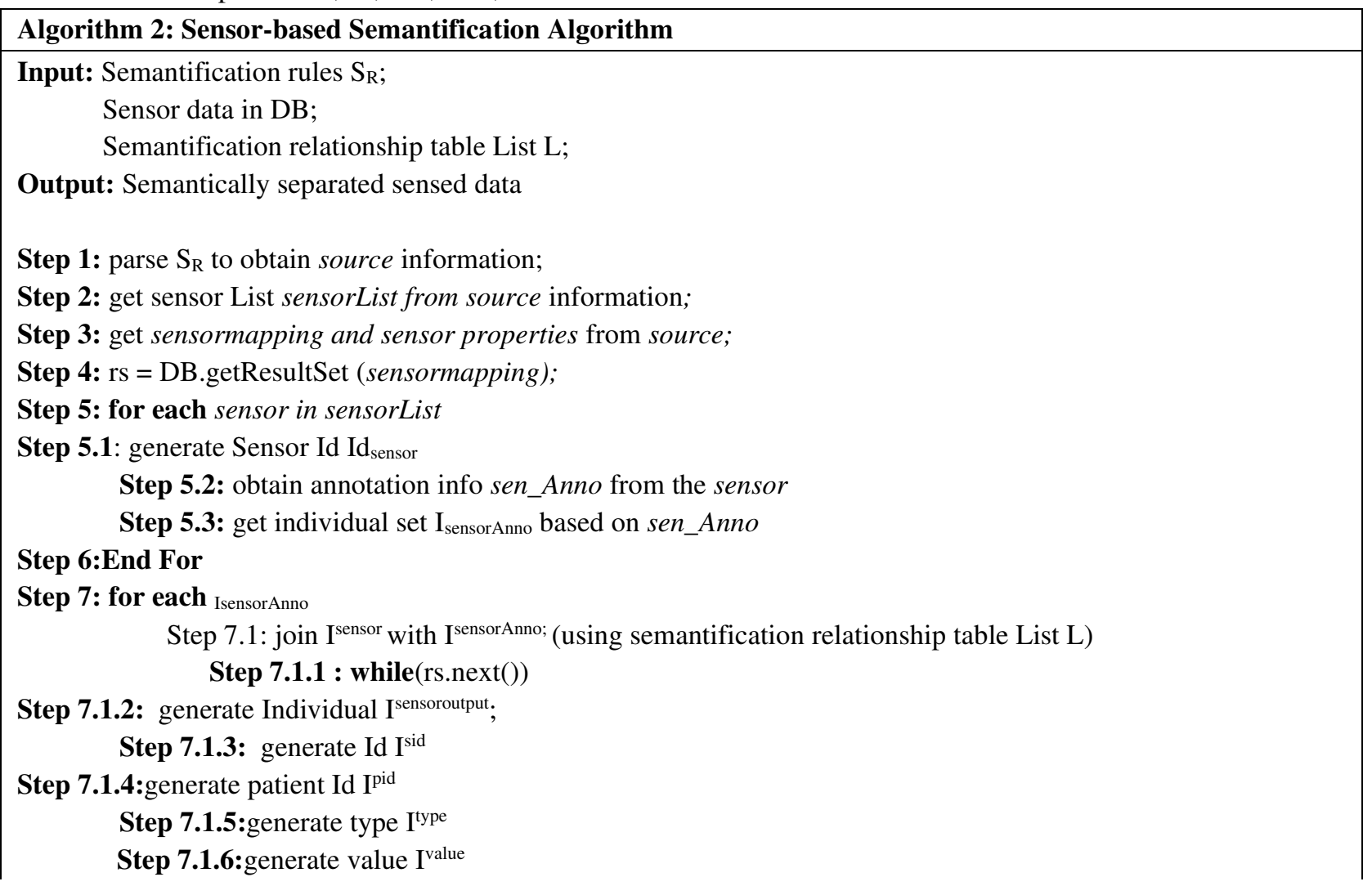


Step 7.1.7:generate units Inits

Step 7.1.8:map I ${ }^{\text {sensoroutput }}$ with $I^{\text {sid }}, I^{\text {pid }}, I^{\text {type }}, I^{\text {value }}, I^{\text {units }}$

Step 7.1.9:map I ${ }^{\text {sensor }}$ with $I^{\text {sensoroutput }}$

End FOR

End WHILE

The sensed objects will now have a meaningful interpretation of which sensor have the associated properties and the disease for which the sensor is being used as shown in the below JSON format as follows.

“@resource_id": "DIA1202",

“@resource_name": “SEN23",

"Disease name:" "Diabetes"

"Pateint_id":"P123",

"Pateint_name":"John",

"data link layer standard":" Ieee 802.15.4",

" date" : "16.07.2019",

"Time_stamp": 10.19.21

"Age":"60"

"Heart Rate": "72",

"Pulse Rate": "78",

"Respiratory Rate": "value”: "18”,

"Blood oxygen level": "88",

\subsection{Semantic similarity mapping algorithm}

All these fetched data are stored as topics in MQTT interface, which acts as a broker, between MQTT publish and MQTT subscribe. Table-II depicts the notations used in the proposed work for implementing the SeSem framework.

Table II Notations and Definition

\begin{tabular}{|l|l|}
\hline Notation & Definition \\
\hline $\mathrm{L}$ & Levenshtein distance \\
\hline Len & length of the string \\
\hline $\mathrm{np}$ & nearest parent \\
\hline $\mathrm{U}$ & Relationship between $\mathrm{P}_{\mathrm{i}}, \mathrm{np}$ \\
\hline $\mathrm{S}_{\mathrm{j}}$ & Subscribed node \\
\hline $\mathrm{p}_{\mathrm{j}}$ & Published node \\
\hline $\mathrm{V}$ & Relationship between $\mathrm{S}_{\mathrm{j}}, \mathrm{np}$ \\
\hline $\mathrm{W}$ & relationship between $\mathrm{S}^{\mathrm{j}}$ and Pi \\
\hline Pos $(\mathrm{n})$ & determine the position of the node in the tree \\
\hline $\mathrm{h}(\mathrm{t})$ & Hidden State \\
\hline $\mathrm{h}(\mathrm{t}-1)$ & Previous Hidden State \\
\hline $\mathrm{x}(\mathrm{t})$ & Input of what pls describe \\
\hline$\theta$ & Parameters Passed to the function $\mathrm{f}$ \\
\hline$A_{i}, A_{j}$ & Semantically separated sensed attributes \\
\hline
\end{tabular}

In MQTT publish, all the sensed data from various health-based sensors are updated to the broker, datastore, after SeSem schema and ontology mapping. Now the end-user, namely doctors, nurses, patient or patient caretaker, can subscribe for a particular physiological parameter, of a particular sensor to know about the current status. Unlike the other existing methodologies, our proposed framework, will response only with the subscribed parameter, avoiding network traffic and lowers energy consumption by the fast response. In order to achieve this condition, our framework 
proposes a semantic similarity matching algorithm, which performs the semantic similarity between the published data and end-user subscription, which will list only the requested physiological parameter, as per a particular date and time. Our proposed healthcare application, allows the end-user to subscribe by giving the following as input patterns $:<<$ patient_name, patient_id, disease_name, date, time (optional)〉>.By applying the similarity algorithm, a JSON file consisting of particular sensor detail, for a particular patient at the specified date and time can be obtained. The performances of the semantification by applying the interoperable semantic similarity matching algorithm for our work are shown in Algorithm 3. The proposed algorithm first standardizes the algorithm, by dividing each entity as tokens and the removes repeated parameters and meaningless words, incomplete and empty words. Next, the published sensed data and user subscribed service requests are analyzed and a hierarchical tree structure is formed.The next step is the calculation of semantic similarity in terms of name, attribute, and the relation between the published and subscribed sensed data. The name-based similarity calculation will be of string type and hence humming distance equation can be applied. In equation (1), L defines the Levenshtein distance between the strings and len represents the length of the string. Among surface similarity, Structural similarity and thematic similarity, thematic similarity will focus on context search as surface and structural similarity, but it will also consider the concepts and their relationships. In order to find the thematic similarity between concepts, linguistic similarity is calculated as in equation (1), as follows:

$S\left(P_{i}, S_{j}\right)=\frac{1}{\frac{L\left(P_{i}, S_{j}\right)}{e^{c C i e n+S j l e n}-L\left(P_{i}, S_{j}\right)}}+\frac{\left(\sum_{x=1}^{\min \left(\left|A_{i}\right|,\left|A_{j}\right|\right){ }} f(x)\right)+\left|A_{i}\right|-\left|A_{j}\right|}{\max \left(\left|A_{i}\right|,\left|A_{j}\right|\right)}+1-D(P i, n p)+D(\mathrm{Sj}, n p)$

shere, $\mathrm{D}\left(\mathrm{P}_{\mathrm{i}}, \mathrm{np}\right)=\sum_{\mathrm{x} \in \mathrm{U}}^{n} \operatorname{Pos}(\mathrm{X})-\sum_{\mathrm{Z} \in \mathrm{W}}^{n} \operatorname{Pos}(\mathrm{Z})$ and $\mathrm{D}\left(\mathrm{S}_{\mathrm{j}}, \mathrm{np}\right)=\sum_{\mathrm{Y} \in \mathrm{V}}^{n} \operatorname{Pos}(\mathrm{Y})-\sum_{\mathrm{Z} \in \mathrm{W}}^{n} \operatorname{Pos}(\mathrm{Z})$

In equation (1), np represents the nearest parent, $U$ represents the relationship between $P_{i} a n d n p, V$ represents the relationship between $S_{j}$ andnp and $\mathrm{W}$ represents the relationship between $\mathrm{S}^{\mathrm{j}}$ and Pi.Pos $(\mathrm{n})$ determines the position of the node in the tree. If there is a matching pair for the set $\left(\mathrm{P}_{\mathrm{i}}, \mathrm{S}_{\mathrm{j}}\right)$, and if it has a child node, keeping the child node as a new set, find out the semantic similarity for the new sets and repeat the procedure, until there is no matching pair.

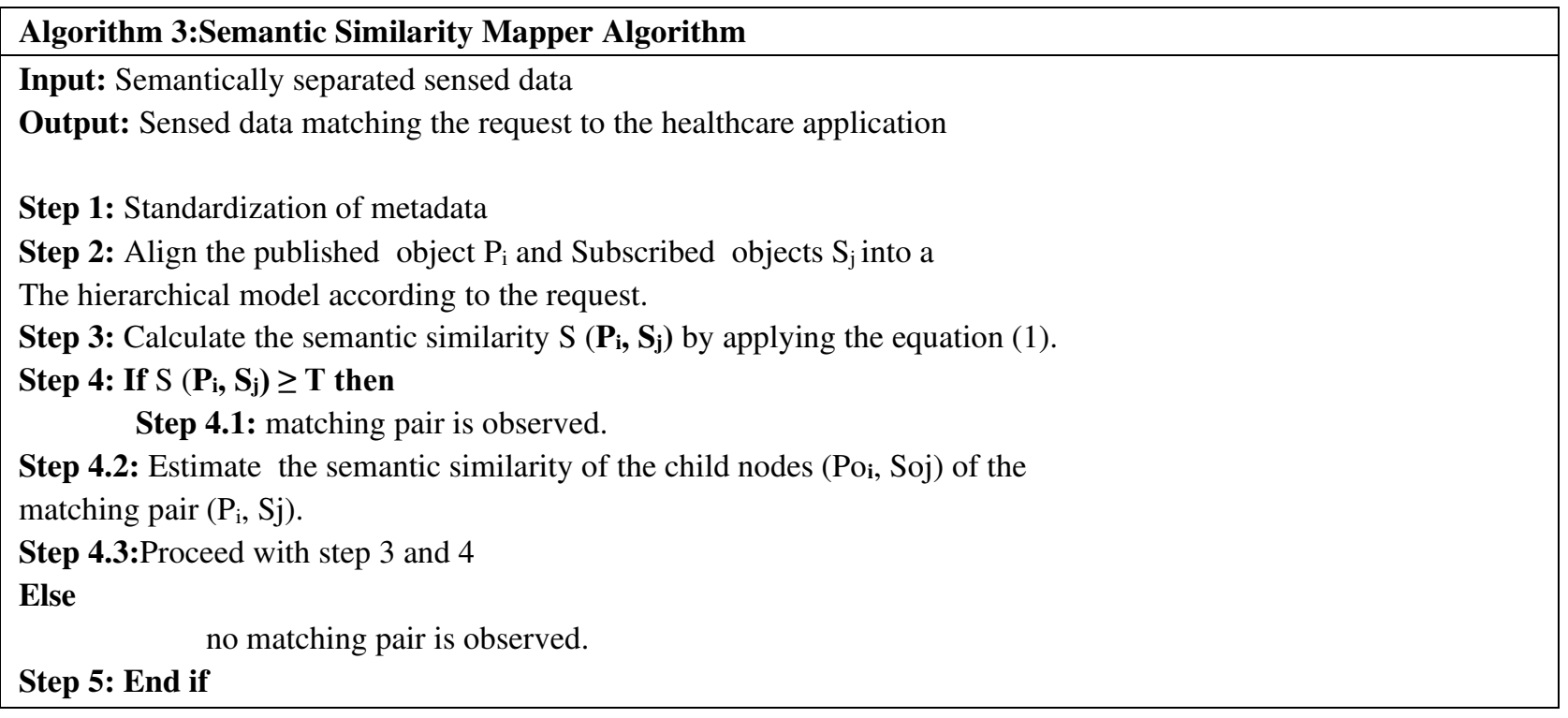

\subsection{Intelligent Service deployments}

To make optimize our proposed system in an intelligent manner, the semantically separated sensor values in JSON format are stored in cloud storage. The end-user of our health care system can obtain a particularphysiological parameter, by subscribing to our framework by issuing a query, containing $:<<$ patient_name, patient_id, 
disease_name, date, time (optional)>>, which was discussed in the above sections. Suppose if a patient didn't specify date and time, the sensor values will be populated in a tremendous amount with a particular physiological parameter for different data and time. Here an intelligent way of responding to the end user's subscription is required, in order to achieve robustness. Hence the fetched semantically separated sensed data are stored in a cloud for applying deep learning algorithms, for achieving intelligent service deployments. Deep learning algorithms are cable of handling millions of data when compared to machine learning algorithms. The training time of deep learning algorithms can be high when compared to machine learning algorithms, but the testing phase will be very quick for deep learning algorithms hence execution time will be pretty less than machine learning algorithms. Our proposed work, utilizes the deep neural network, as there can be any number of hidden layers, for prediction the response in an intelligent way. As our dataset is dependent on time-dependent, Recurrent Neural Network (RNN) algorithms have been used, for predicting the sensed values based on time and priority. Recurrent Neural Network (RNN), learns and predicts based on training, but it does have the unique feature of remembering past values and applying those past values to the next hidden layers, for the prediction of better output. But a Feed-Forward Neural Network does not remember the past as RNN does. Figure 3 explains about RNN. The inputs $\mathrm{X} 1, \mathrm{X} 2, \mathrm{X} 3, \mathrm{X} 4$ refers to our dataset's physiological parameters namely, blood pressure, heart rate, pulse rate, and oxygen rate. The RNN is trained with these 4 inputs and is propagated forward to the hidden layers by applying complex functions. Each layer is updated with the previous layers values and finally reaches the output layer with the predicted output.

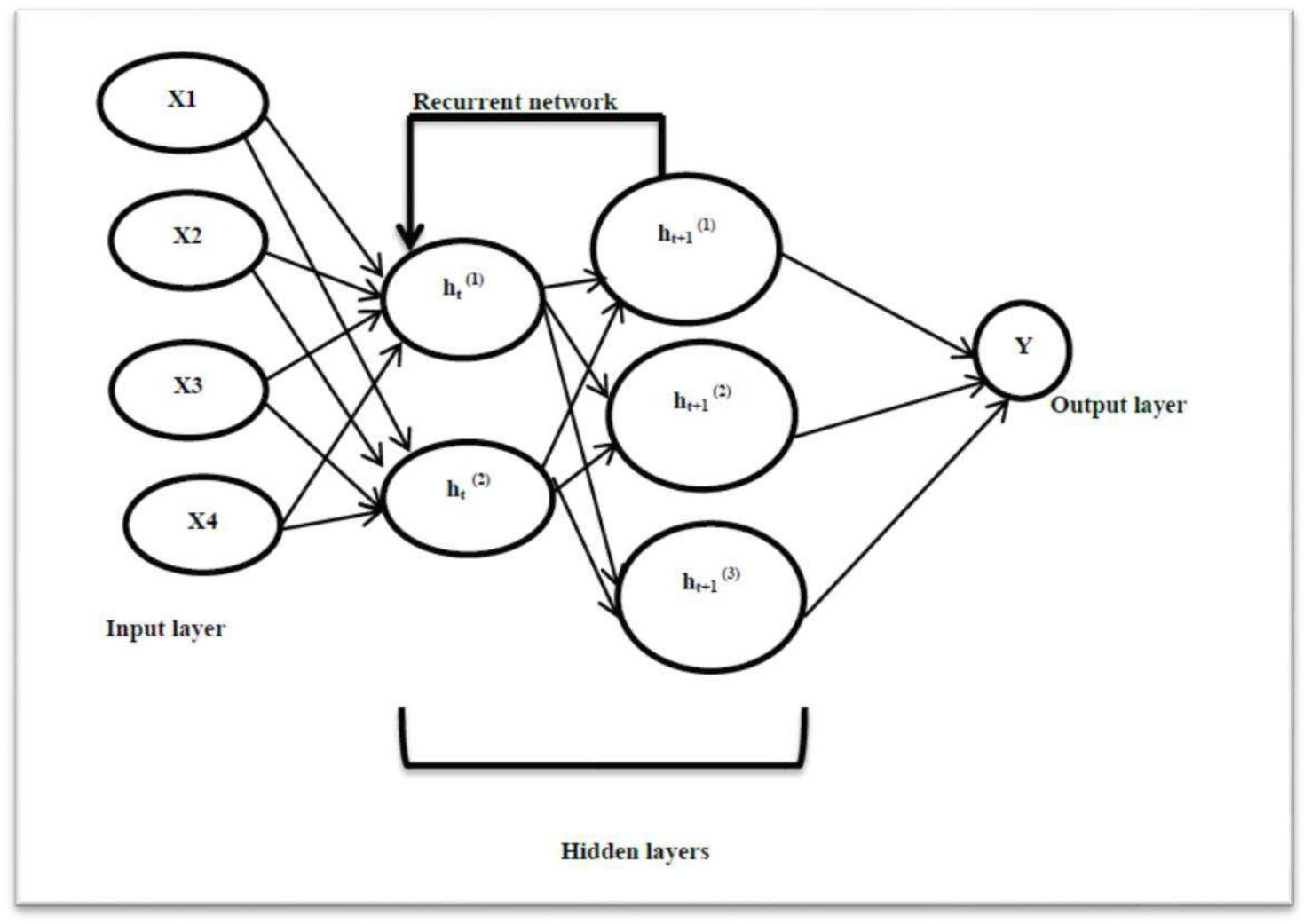

The basic formula of RNN is shown below:

Figure 3. RNN network

$$
\mathrm{h}^{(\mathrm{t})}=\mathrm{f}\left(\mathrm{h}^{(\mathrm{t}-1)}, \mathrm{x}_{\mathrm{i}}{ }^{(\mathrm{t})} ; \theta\right) \quad \text {, where } \mathrm{i}=1 \text { to } 4
$$

It principally states the present hidden state $\mathbf{h}(\mathbf{t})$ is a function $\mathbf{f}$ of the previously hidden state $\mathbf{h}(\mathbf{t}-\mathbf{1})$ and the current input $\mathbf{x}(\mathbf{t})$. The $\theta$ are the parameters of the function $\mathrm{f}$. The network usually learns to use $\mathrm{h}(\mathrm{t})$ as a kind of lossy summary of the task-relevant traits of the past sequence of inputs up to t. 
In our proposed health care system if an end-user subscribes for a particular physiological parameter, without specifying date and time, the deep Recursive Feed Forward Neural Network (RNN), will response with the sensor value, with the latest update. Thus our proposed system can be enhanced and optimized, by applying deep learning algorithms, which helps in an intelligent way of deploying services to the end-users. Algorithm 4 depicts intelligent service deployments as discussed in the above section. Table-2 depicts the meaning of the notations used in algorithms.

As in algorithm 4, the RNN is fed with the semantically separated sensed values. RNN trains the dataset based on the last updated date and time. If an end-user request or a particular physiological parameter with any data and time, RNN trains the parameter's data and time column with last updated value and stores in the registry. From the registry, the service is accomplished with the last updated physiological parameter. Using RNN, we can set a minimum and maximum threshold for every sensed physiological parameter. If a particular physiological parameter exceeds the threshold, doctors will be updated with an alert history, which will contain all the other sensed values at which the physiological parameter under consideration, has exceeded the maximum limit along with data and time. By using this alert message, the doctors can make a decision of disease type and respective treatments immediately.

\section{Algorithm 4: Intelligent service deployment Algorithm \\ Input: Semantically separated sensed data with priority. \\ Output: Last updated sensed data.}

Step 1: load the intended dataset, load (data.json) with subscribed physiological parameter.

Step 2: Apply deep feedforward recursive neural network algorithm.

Step 2.1: If the termination condition is met, terminate the training process

Else

Step 2.2: The algorithm runs by applying equation (2).

Step 2.3: The $\theta$ in the equation(2) is substituted by Last Updated date and time.

End if

\section{Performance evaluation}

The proposed SeSem framework is implemented and tested on Anaconda navigator using the python shell with a 1.2GHz CPU and 1GB SDRAM.Our proposed work was evaluated using the pulseoximator dataset [40], which is the data collected from patients admitted during their ill-periods at the Beth Israel Deaconess Medical Centre (Boston, MA, USA). The physiological parameters, such as blood pressure, heart rate, oxygen saturation, and pulse rate are recorded in 53 recordings with an 8-minute duration for every patient. The experimental results executed by our SeSem framework are tested against the existing methodologies namely NN-DEGI-BP and TRiNF. Figure 5-6 depicts the best value obtained from Positive Predictive value (PPV) and Negative Predictive Value (NPV) in terms of criticality of physiological parameters under concern for NN-DEGI-BP, TRiNF, and SeSem (proposed) methodologies. PPV is a measure of correctly predicting the critical physiological parameters namely blood pressure, oxygen saturation, and heart rate and pulse rate analyzed for a particular patient with respect to his number of stays. Alternatively, NPV is a measure of incorrectly predicting critical signs in the physiological parameters under consideration. We can apply PPV and NPV for assessing moderate and normal heath related parameters also. Figure 4 and 5 depict PPV and NPV for critical condition. From the figures, it is noted that PPV for our proposed SeSem framework has shown the best value of $49 \%$ of PPV and 58\% NPV, proving our proposed framework efficiently predicts the positive and negative scenario of both the disease in an exact manner than NN-DEGI-BP and TRiNF. 


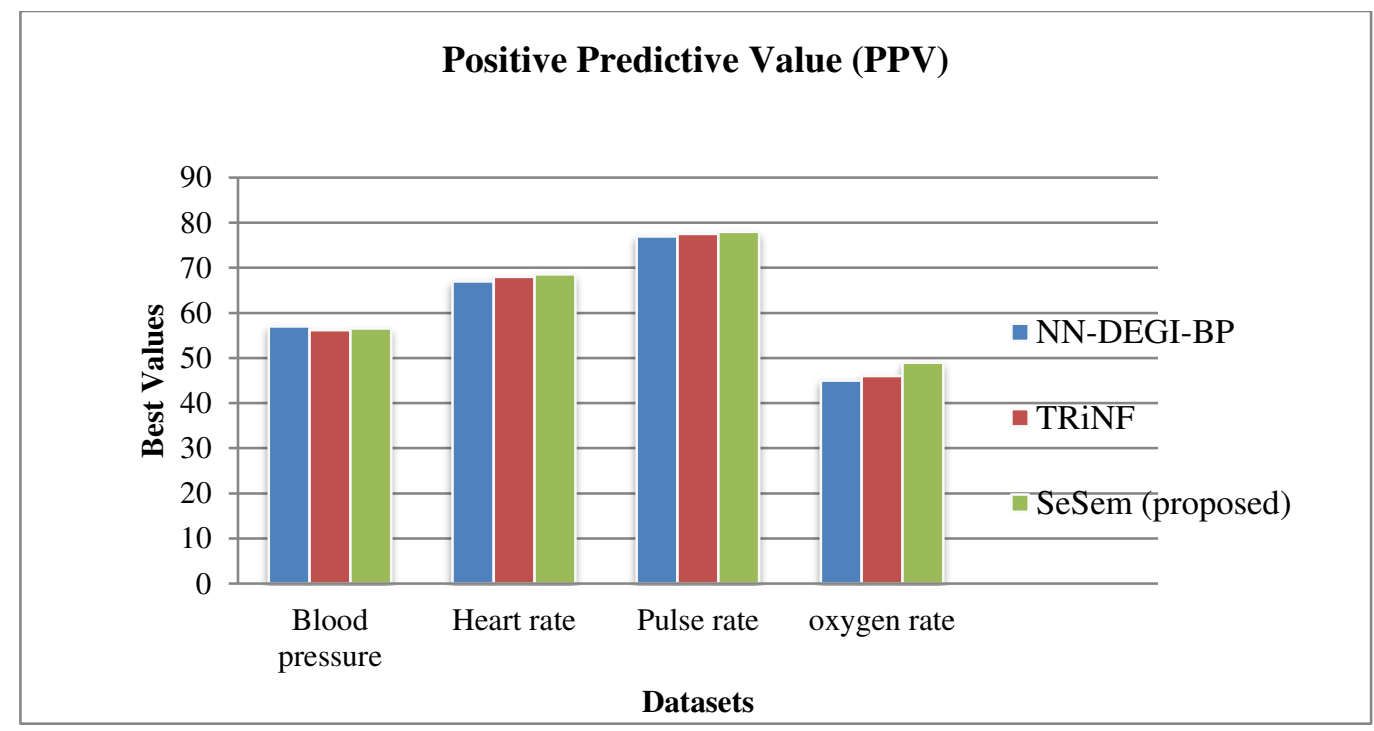

Figure 4. Best PPV Value

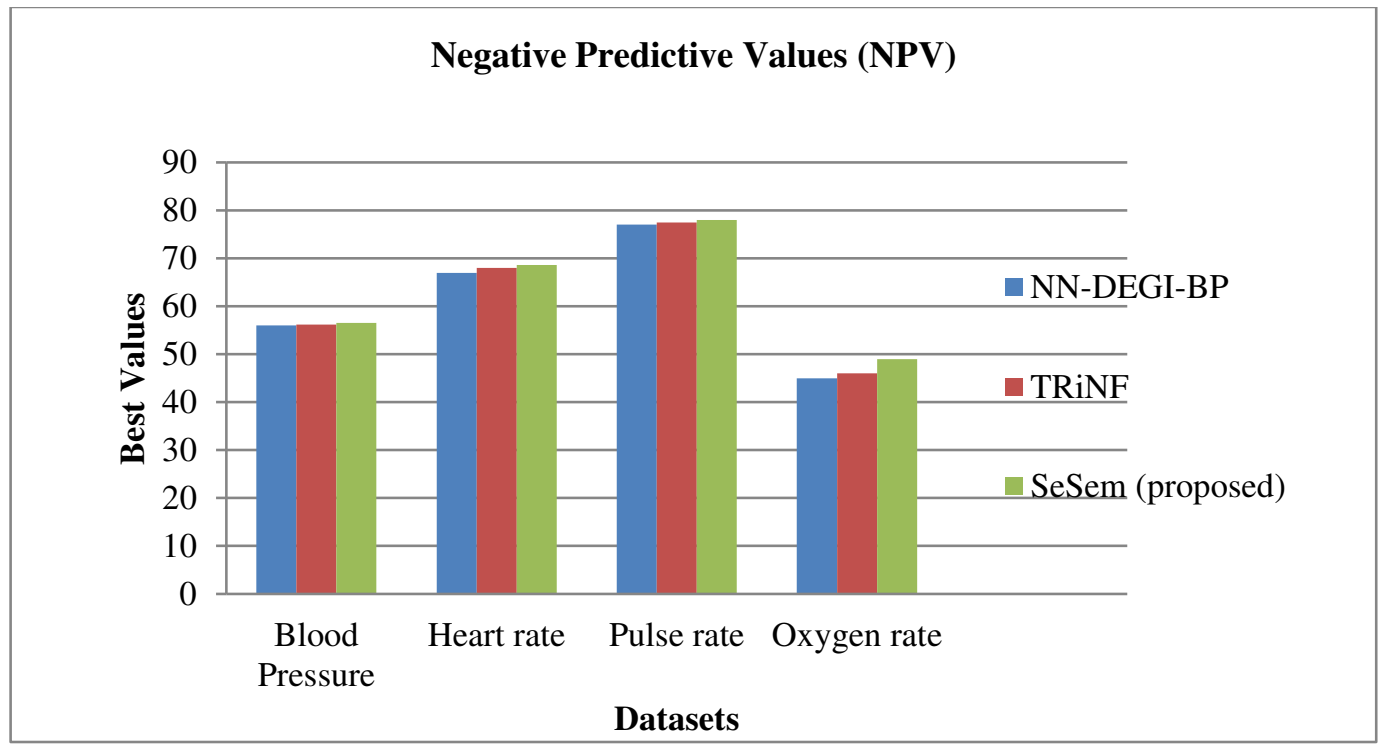

Figures 5. Best NPV Value

Figure 6 compares the classifier accuracy for predicting critical signs in blood pressure, heart rate, pulse rate and oxygen rate in the pulseoximator dataset using NN-DEGI-BP, TRiNF and SeSem framework. The classifier accuracy for our proposed framework is more when compared to that of existing methodologies. For instance, if we take hepatitis,NN-DEGI-BP is 56\%, TRiNF is $78 \%$ ad SeSem framework is $82.3 \%$. Figure 7 compares the classifier accuracy of our proposed SeSem framework with semantification and without semantification. It is found that the SeSem framework has the least accuracy when semantification is not done, but when the sensed data is semantically computed, the classifier prediction accuracy is high, as the dataset will be having a meaningful interpretation. 


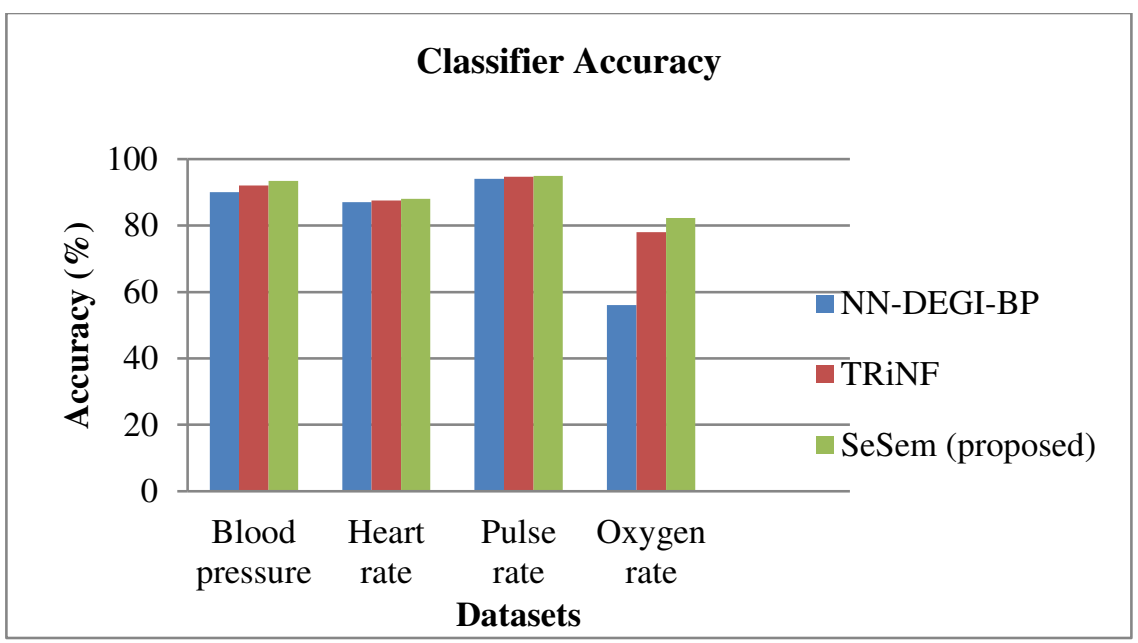

Figure 6. Classifier accuracy for NN-DEGI-BP, TRiNF, and SeSem (proposed)

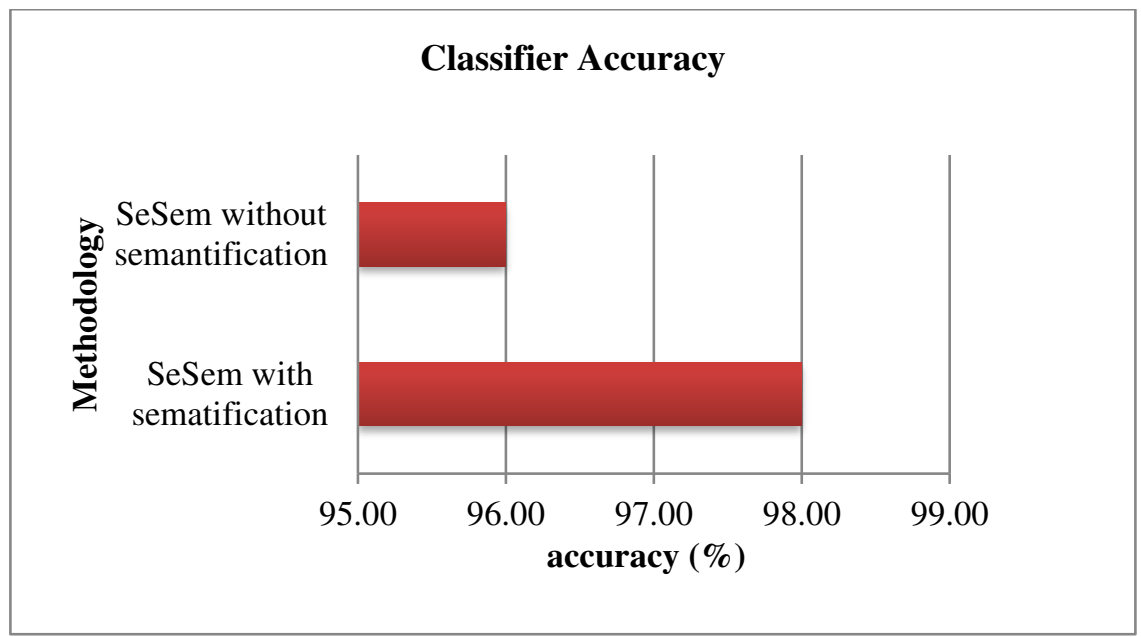

Figure 7. Classifier accuracy with and without semantification

Reliability refers to how well the IoT service is being offered at varying real-world queries and conditions. Figure 8 compares the reliability of two-time series based methods TRiNF and SeSem framework (proposed) against hepatitis and thrombosis datasets. TRiNF has the least reliability of $89 \%$ when compared to that of the SeSem framework with $96 \%$ reliability.

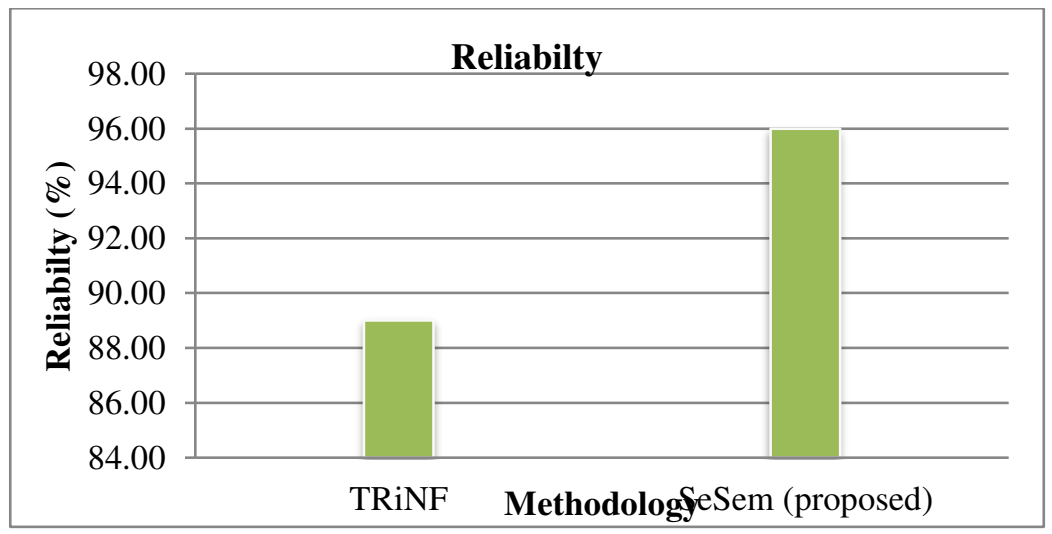

Figure 8. Reliability 
Figure 9 compares the execution time of SeSem framework with the SeCoG framework. It is found that our proposed framework with the semantification process and MQTT protocol has the highest execution time when compared to $\mathrm{SeCoG}$ which uses CoAP for message communication between IoT devices and the Internet. SeSem framework took $341 \mathrm{~ms}$ for executing 10 differentrequestswhereasthe SeCoG framework took $578 \mathrm{~ms}$ for exaction under the same scenario. This shows that our SeSem framework offers user-friendly services with better performance when compared to that of other existing methodologies, using semantification and MQTT protocol.

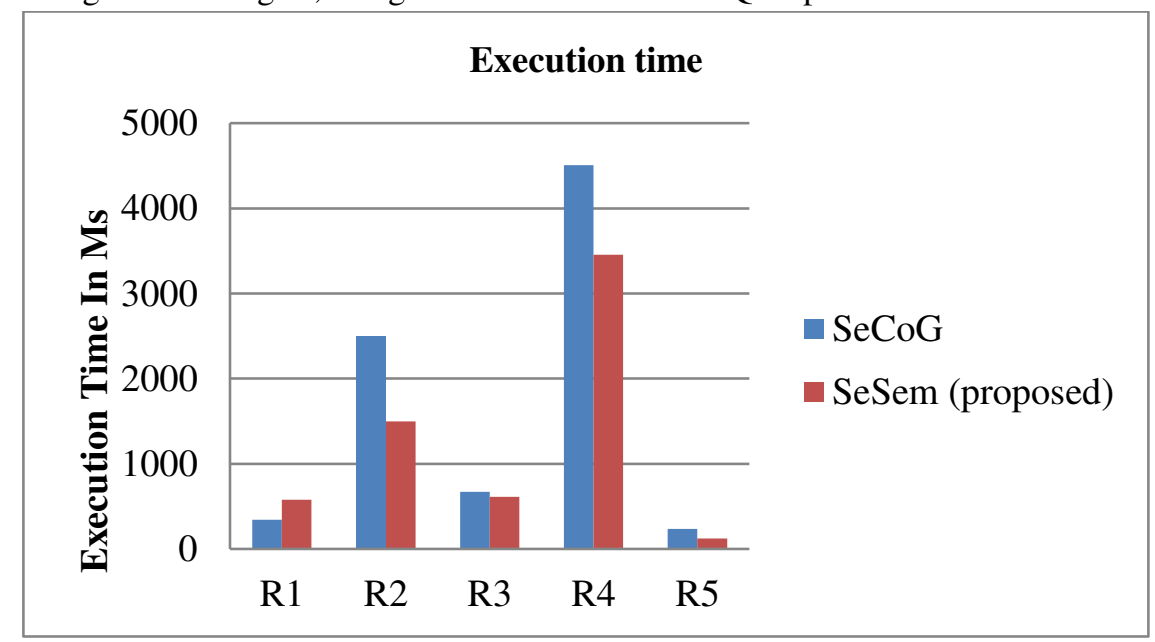

Figure 9. Execution time

Figure 10 depicts the misclassification percentage between training and testing data considering the features in the pulse oximeter dataset. The misclassification rate predicts the percentage of false predictions made against true predictions by comparing the training and testing data. It is found that the proposed SeSem framework has the least misclassification percentage when compared to other existing methodologies.

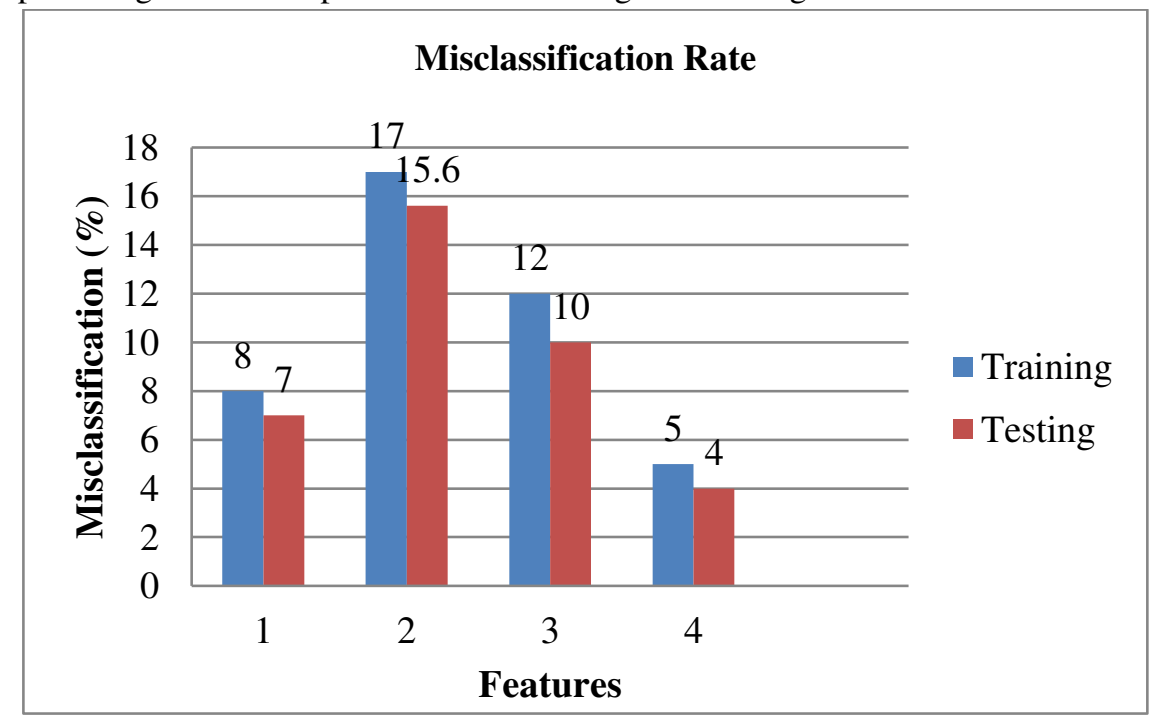

Figure 10. Misclassification Rate

From the performance evaluation, it is found that our proposed SeSem framework excels the performance metric in terms of PPV, NPV, accuracy, reliability, execution time and misclassification rate when compared to that TRiNF and NN-DEGI-BP. Our proposed has proved to be the best framework for IoT user-specific MQTT based services as each and every sensed data are semantically separated and mapped with the user's specific service request using semantic mapper algorithm in order to fulfill the appropriate requested service. 


\section{Conclusion}

In order to promote a robustness, efficient and intelligible health care system, the proposed Sensor-based Semantification (SeSem) framework, which works semantification based on priority by applying semantification rules and semantification relation table, in order to separate the sensed JSON data into a meaningful format. Sensor-based Semantic (SS) ontology is applied to provide meaningful service with respect to the sensors and diseases for which the sensor is used. For user-specific service requisite, a semantic similarity mapping algorithm, semantic similarity mapper is proposed and implemented, which offers services based on the subscribed physiological parameters. If an end-user failed to provide date and time for the subscribed sensor data, a deep recursive feed-forward neural network algorithm is applied to the collected subscriber-specific sensor data. The deep learning predicts only the subscribed sensor data with the last updated date and time and services the end-user with the updated medical record. The RNN also updates the doctors with an alert message of when a particular physiological parameter, exceeds the minimummaximum threshold along with date and time. The proposed methodology is evaluated against IoT performance metrics namely Positive Predictive value (PPV), Negative Predictive Value (NPV), accuracy, reliability, execution time and misclassification rate showing better results in terms of service-oriented IoT. Thus our proposed system achieves a user-specific intelligent health care system, with robustness and efficiency when compared to the existing healthcare systems. Work is being carried out to compare the performance of SeSem framework when CoAP is used as communication protocol and Multi-layered Perceptrons (MLP's) is used as a classifier, to deploy user's service request intelligently.

\section{DECLARATION STATEMENTS:}

Funding: No Funding for this work is allotted.

Conflicts of interest: No confilt of Intrest.

Availability of data and material: Private Data.

Code availability: Coustomed code to achieve efficiency.

\section{AUTHOR CONTRIBUTIONS}

Conceptualization: R.R, A.B and G.K. Methodology: R.R.,A.B\&G.K. Software: R.R, A.B and G.K Validation: R.R, A.B andG.K. Formal analysis: R.R. Investigation: R.R,A.B and G.K. Resources: R.R. Writing-original draft preparation:R.R. Writing-review and editing: R.R,A.B and G.K. Supervision: R.R.,A.B and G.K.

\section{References}

1. $\mathrm{Da} \mathrm{Xu}, \mathrm{Li}, \mathrm{Wu} \mathrm{He}$, and Shancang Li. "Internet of things in industries: A survey." IEEE Transactions on industrial informatics 10, no. 4 (2014): 2233-2243.

2. Shi, Yulong, Yang Zhang, Hans-Arno Jacobsen, Lulu Tang, Geoffrey Elliott, Guanqun Zhang, Xiwei Chen, and Junliang Chen. "Using Machine Learning to Provide Reliable Differentiated Services for IoT in SDNLike Publish/Subscribe Middleware." Sensors 19, no. 6 (2019): 1449.

3. Jia, Bing, Lifei Hao, Chuxuan Zhang, Huili Zhao, and Muhammad Khan. "An IoT service aggregation method based on dynamic planning for QoE restraints." Mobile Networks and Applications 24, no. 1 (2019): 25-33.

4. RavitejaBalekai, Prof. Raghudathesh G P, Megha D H, Bindu H V, Madhuri C N."MQTT Based Patient Health Monitoring". International Journal of Pure and Applied Mathematics, Volume 120 No. 6 (2018), 799807. 
5. P. C. Calcina-Ccori, L. C. C. De Biase, G. Fedrecheski, F. S. Corrêa da Silva and M. K. Zuffo, "Enabling Semantic Discovery in the Swarm," in IEEE Transactions on Consumer Electronics, vol. 65, no. 1, pp. 5763, Feb. 2019.

6. H.M. Hasan, S.A.Jawad.'IoT Protocols for Health Care Systems: A Comparative Study".IJCSMC, Vol. 7, Issue. 11, November (2018), pg.38-4.

7. Mishra, Ayaskanta, AkankshaKumari, PoojaSajit, and PranjalPandey. "Remote web-based ECG Monitoring using MQTT Protocol for IoT in Healthcare." Development 5, no. 04 (2018).

8. Ismail, Lina Nachabe, Marc Girod-Genet, and Bachar El Hassan. "Semantic techniques for IoT data and service management: ONTOSMART system." International journal of wireless \& mobile networks (IJWMN) 8, no. 4 (2016): 43-63.

9. Cirani, Simone, Luca Davoli, Gianluigi Ferrari, RémyLéone, Paolo Medagliani, Marco Picone, and Luca Veltri. "A scalable and self-configuring architecture for service discovery in the internet of things." IEEE Internet of Things Journal 1, no. 5 (2014): 508-521.

10. Kim, Meesun, Kyoungsook Kim, KyongduckSeo, Joosang Lee, Kyoungsik Park, and Kwanghoon Kim. "Modeling process-aware Internet of Things services over an ARDUINO community computing environment." In 2017 19th International Conference on Advanced Communication Technology (ICACT), pp. 163-166. IEEE, 2017.

11. Davoli, Luca, MattiaAntonini, and Gianluigi Ferrari. "DiRPL: A RPL-Based Resource and Service Discovery Algorithm for 6LoWPANs." Applied Sciences 9, no. 1 (2019): 33.

12. Lu, Ching-Hu, and Chia-En Tsai. "IoT-Enabled Cross-Field and Reconfigurable Service Provisioning With User-Centered Design." IEEE Systems Journal (2019).

13. Kousiouris, George, StylianosTsarsitalidis, EvangelosPsomakelis, Stavros Koloniaris, Cleopatra Bardaki, Konstantinos Tserpes, Mara Nikolaidou, and Dimosthenis Anagnostopoulos. "A microservice-based framework for integrating IoT management platforms, semantic and AI services for the supply chain management." ICT Express 5, no. 2 (2019): 141-145.

14. Zhang, Xiang, Lina Yao, Chaoran Huang, Sen Wang, Mingkui Tan, Guodong Long, and Can Wang. "Multimodality sensor data classification with selective attention." arXiv preprint arXiv:1804.05493 (2018).

15. Jean Paul, Bambanza. "iSEE: A Semantic Sensors Selection System for Healthcare." (2016).

16. Dautov, Rustem, Salvatore Distefano, and Rajkumaar Buyya. "Hierarchical data fusion for Smart Healthcare." Journal of Big Data 6, no. 1 (2019): 19.

17. Rhayem, Ahlem, Mohamed Ben Ahmed Mhiri, Mayssa Ben Salah, and Faiez Gargouri. "Ontology-based system for patient monitoring with connected objects." Procedia Computer Science 112 (2017): 683-692.

18. Honti, GergelyMarcell, and Janos Abonyi. "A Review of Semantic Sensor Technologies in the Internet of Things Architectures." Complexity 2019 (2019).

19. Alamri, Abdullah. "Ontology Middleware for Integration of IoT Healthcare Information Systems in EHR Systems." Computers 7, no. 4 (2018): 51.

20. Mavrogiorgou, Argyro, Athanasios Kiourtis, Konstantinos Perakis, Stamatios Pitsios, and Dimosthenis Kyriazis. "IoT in Healthcare: Achieving Interoperability of High-Quality Data Acquired by IoT Medical Devices." Sensors 19, no. 9 (2019): 1978.

21. Honti, GergelyMarcell, and Janos Abonyi. "A Review of Semantic Sensor Technologies in the Internet of Things Architectures." Complexity 2019 (2019).

22. TsiachriRenta, Pelagia, SteliosSotiriadis, and Euripides GM Petrakis. "Healthcare sensor data management on the cloud." In Proceedings of the 2017 Workshop on Adaptive Resource Management and Scheduling for Cloud Computing, pp. 25-30. ACM, 2017.

23. Mavrogiorgou, Argyro, Athanasios Kiourtis, Konstantinos Perakis, Stamatios Pitsios, and Dimosthenis Kyriazis. "IoT in Healthcare: Achieving Interoperability of High-Quality Data Acquired by IoT Medical Devices." Sensors 19, no. 9 (2019): 1978.

24. Echeverría, Matías, Angel Jimenez-Molina, and Sebastián A. Ríos. "A semantic framework for continuous u-health services provisioning." Procedia Computer Science 60 (2015): 603-612. 
25. Rodríguez-Molina, Jesús, José-FernánMartínez, Pedro Castillejo, and Lourdes López. "Combining wireless sensor networks and semantic middleware for an internet of things-based sportsman/woman monitoring application." Sensors 13, no. 2 (2013): 1787-1835.

26. Ajigboye, Olamidipupo Solomon. "Conceptual Framework for Semantic Interoperability in Sensor-enhanced Health Information Systems (SIOp4Se-HIS)." Ph.D. diss., Kingston University, 2018.

27. Sarierao, Borade Samar, and Amara Prakasarao. "Smart Healthcare Monitoring System Using MQTT Protocol." In 2018 3rd International Conference for Convergence in Technology (I2CT), pp. 1-5. IEEE, 2018.

28. Kumar, J. Dr. S. Mohan, and DarpanMajumder. "Healthcare Solution based on Machine Learning Applications in IoT and Edge Computing." International Journal of Pure and Applied Mathematics 119, no. 16 (2018): 1473-1484.

29. Edoh, Thierry. "Internet of things in emergency medical care and services." In Medical Internet of Things (m-IoT)-Enabling Technologies and Emerging Applications. IntechOpen, 2019.

30. Salam, Abdul, Adnan Nadeem, Kamran Ahsan, Muhammad Sarim, and KashifRizwan. "A Novel QoS algorithm for Healthcare Applications of Body Area Sensor Networks." published in textroad Journal of Basic and Applied Scientific Research 4, no. 1 (2014): 169-178.

31. Abidoye, Ademola P., Nureni A. Azeez, Ademola OlusolaAdesina, and Kehinde K. Agbele. "Using wearable sensors for the remote healthcare monitoring system." (2011).

32. Gambhir, Sapna, and MadhumitaKathuria. "DWBAN: Dynamic priority based WBAN architecture for healthcare system." In 2016 3rd International Conference on Computing for Sustainable Global Development (INDIACom), pp. 3380-3386. IEEE, 2016.

33. Dias, Duarte, and João Paulo Silva Cunha. "Wearable health devices—vital sign monitoring, systems and technologies." Sensors 18, no. 8 (2018): 2414.

34. Albahri, O. S., A. S. Albahri, K. I. Mohammed, A. A. Zaidan, B. B. Zaidan, M. Hashim, and Omar H. Salman. "Systematic review of real-time remote health monitoring system in triage and priority-based sensor technology: Taxonomy, open challenges, motivation and recommendations." Journal of medical systems 42, no. 5 (2018): 80.

35. Wan, Jie, Munassar AAH Al-awlaqi, MingSong Li, Michael O'Grady, Xiang Gu, Jin Wang, and Ning Cao. "Wearable IoT enabled real-time health monitoring system." EURASIP Journal on Wireless Communications and Networking 2018, no. 1 (2018): 298.

36. Tang, Jie, Dawei Sun, Shaoshan Liu, and Jean-Luc Gaudiot. "Enabling deep learning on IoT devices." Computer 50, no. 10 (2017): 92-96.

37. Noura, Mahda, Mohammed Atiquzzaman, and Martin Gaedke. "Interoperability in the internet of things: Taxonomies and open challenges." Mobile Networks and Applications 24, no. 3 (2019): 796-809.

38. Jane, Nancy Yesudhas, KhannaHarichandran Nehemiah, and Kannan Arputharaj. "A temporal mining framework for classifying un-evenly spaced clinical data." Applied clinical informatics 7, no. 01 (2016): 121.

39. Leema, N., H. Khanna Nehemiah, and Arputharaj Kannan. "Neural network classifier optimization using differential evolution with global information and backpropagation algorithm for clinical datasets." Applied Soft Computing 49 (2016): 834-844.

40. Valliyammai. C and Bhuvaneswari A, "Semantics-based sensitive topic diffusion detection framework towards privacy aware Online Social Networks", Cluster Computing, 22(1), Springer, pp. 1-16, 2018.

41. Bhuvaneswari, A and Valliyammai, C, 'Semantic-based sensitive topic dissemination control mechanism for safe social networking', Rajsingh, E.B, et al. (eds.), Advances in Big Data and Cloud Computing, Advances in Intelligent Systems and Computing, Volume 645, Chapter No. 17, pp. 197-207. Springer Nature, Singapore, 2018.

42. Bhuvaneswari, A and Valliyammai, C, 'Social IoT enabled emergency event detection framework using geo tagged microblogs and crowdsourced photos', Abraham, A, et al. (eds.), Emerging Technologies in Data Mining and Information Security, Advances in Intelligent Systems and Computing, Volume 813, Chapter No. 13, pp. 151-162. Springer Nature, Singapore, 2018. 
43. Pimentel, M.A.F. et al. Towards a Robust Estimation of Respiratory Rate from Pulse Oximeters. IEEE Transactions on Biomedical Engineering, 64(8), pp.1914-1923, $2016 . \quad$ [DOI: 10.1109/TBME.2016.2613124](http://doi.org/10.1109/TBME.2016.2613124).

44. Goldberger AL, Amaral LAN, Glass L, Hausdorff JM, Ivanov PCh, Mark RG, Mietus JE, Moody GB, Peng C-K, Stanley HE. PhysioBank, PhysioToolkit, and PhysioNet: Components of a New Research Resource for Complex Physiologic Signals (2003). Circulation. 101(23):e215-e220. 


\section{Figures}

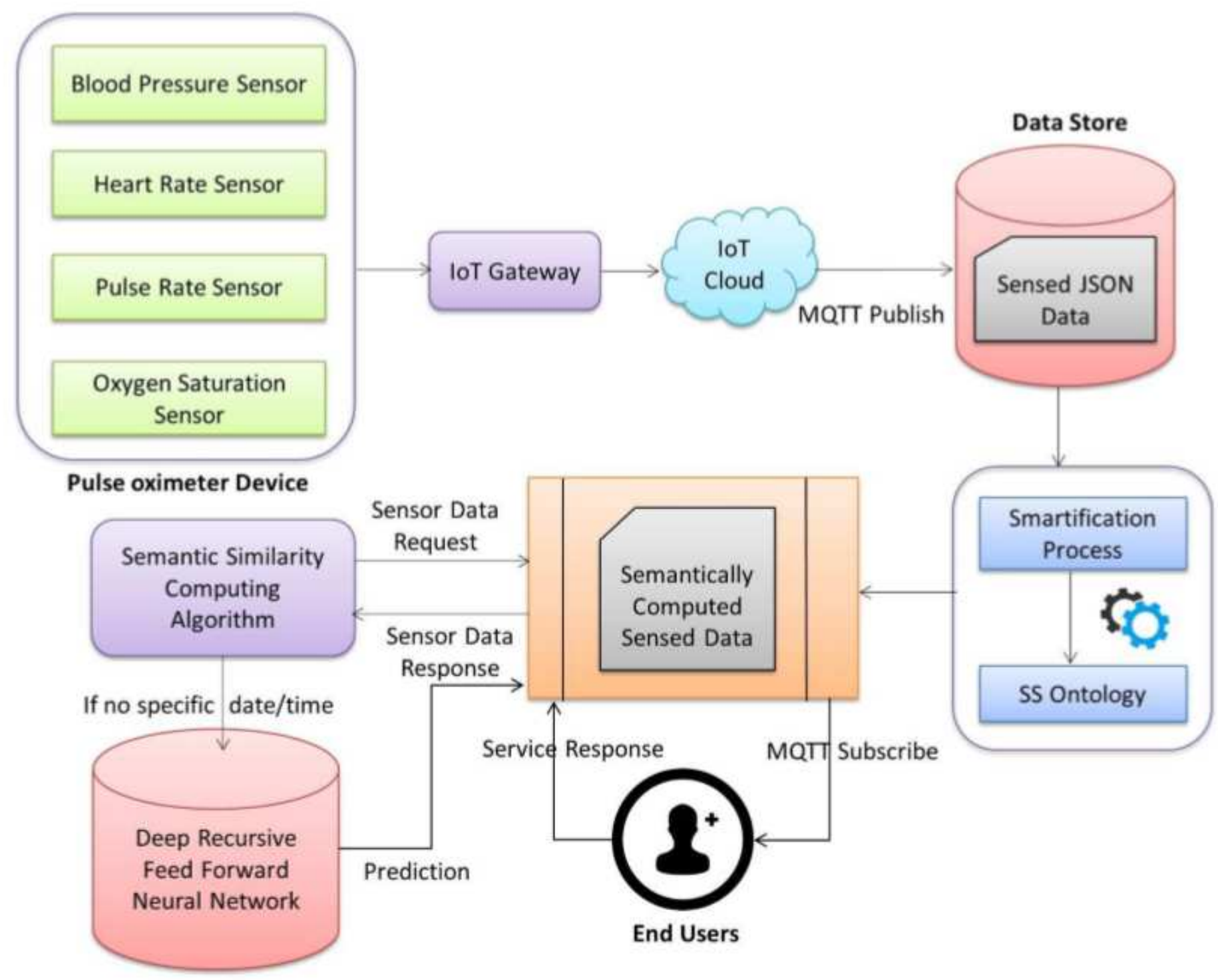

Figure 1

Proposed SeSem Health care system 


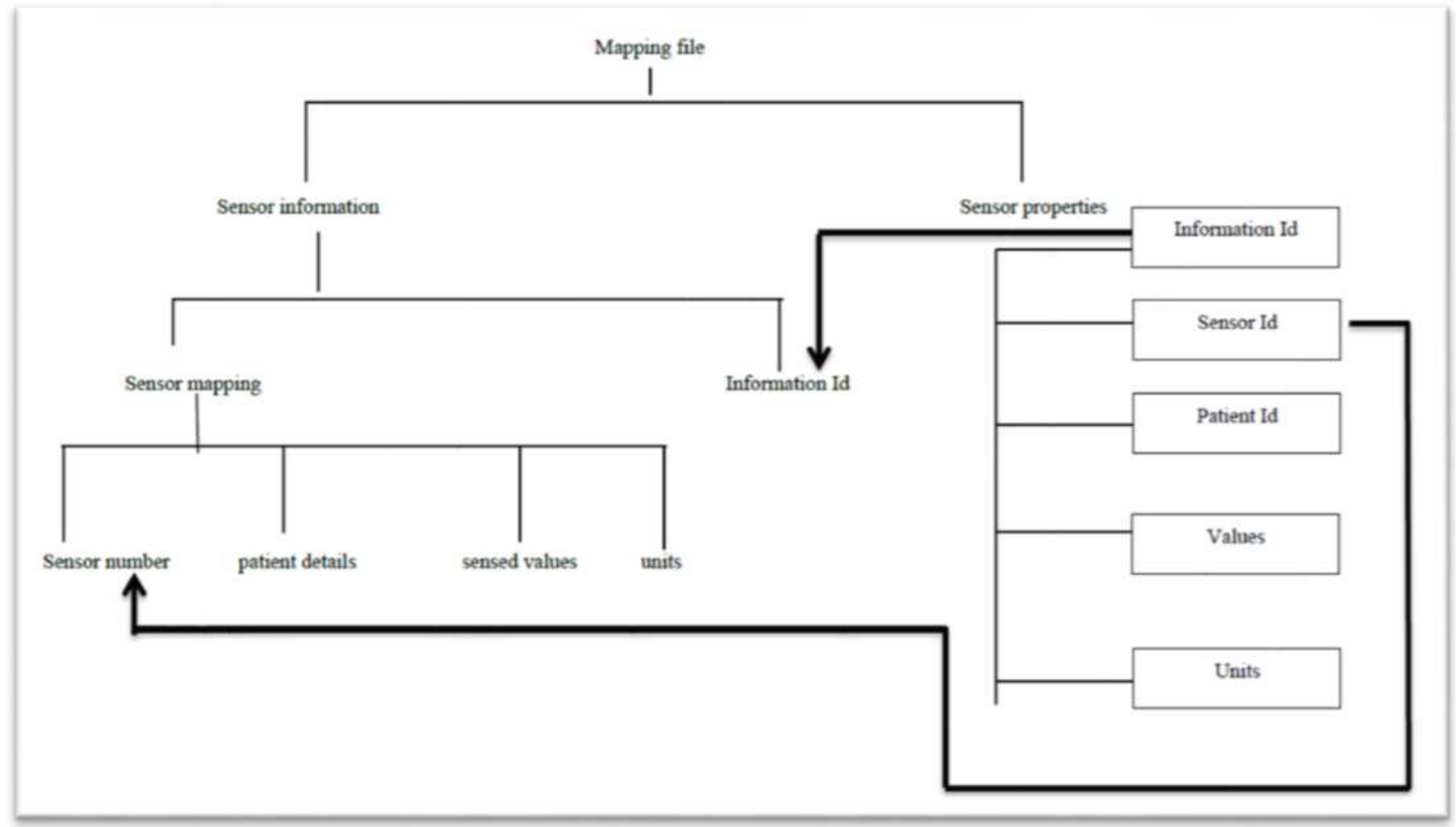

Figure 2

Sematification rules 


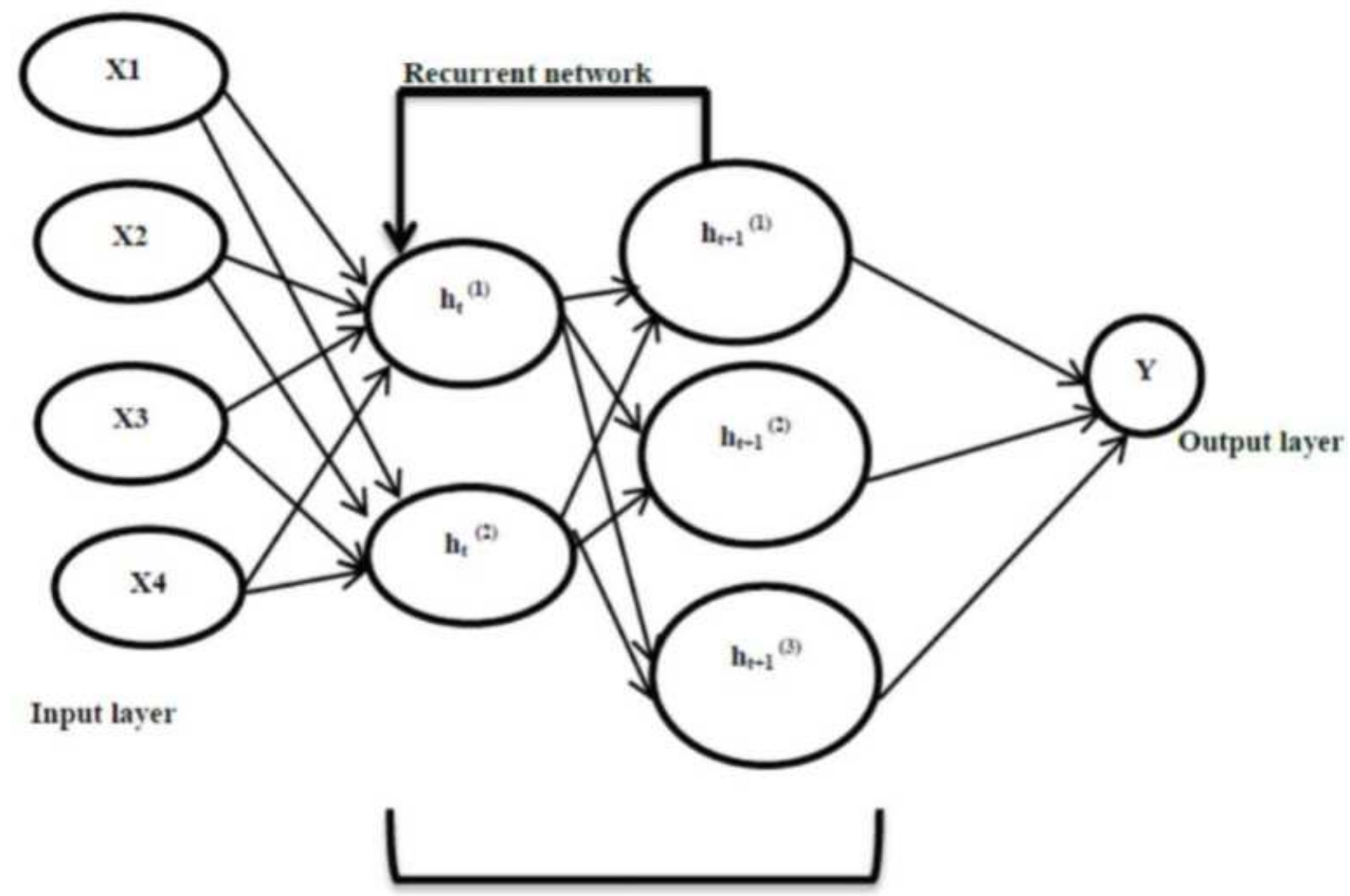

Hidden layers

Figure 3

RNN network 


\section{Positive Predictive Value (PPV)}

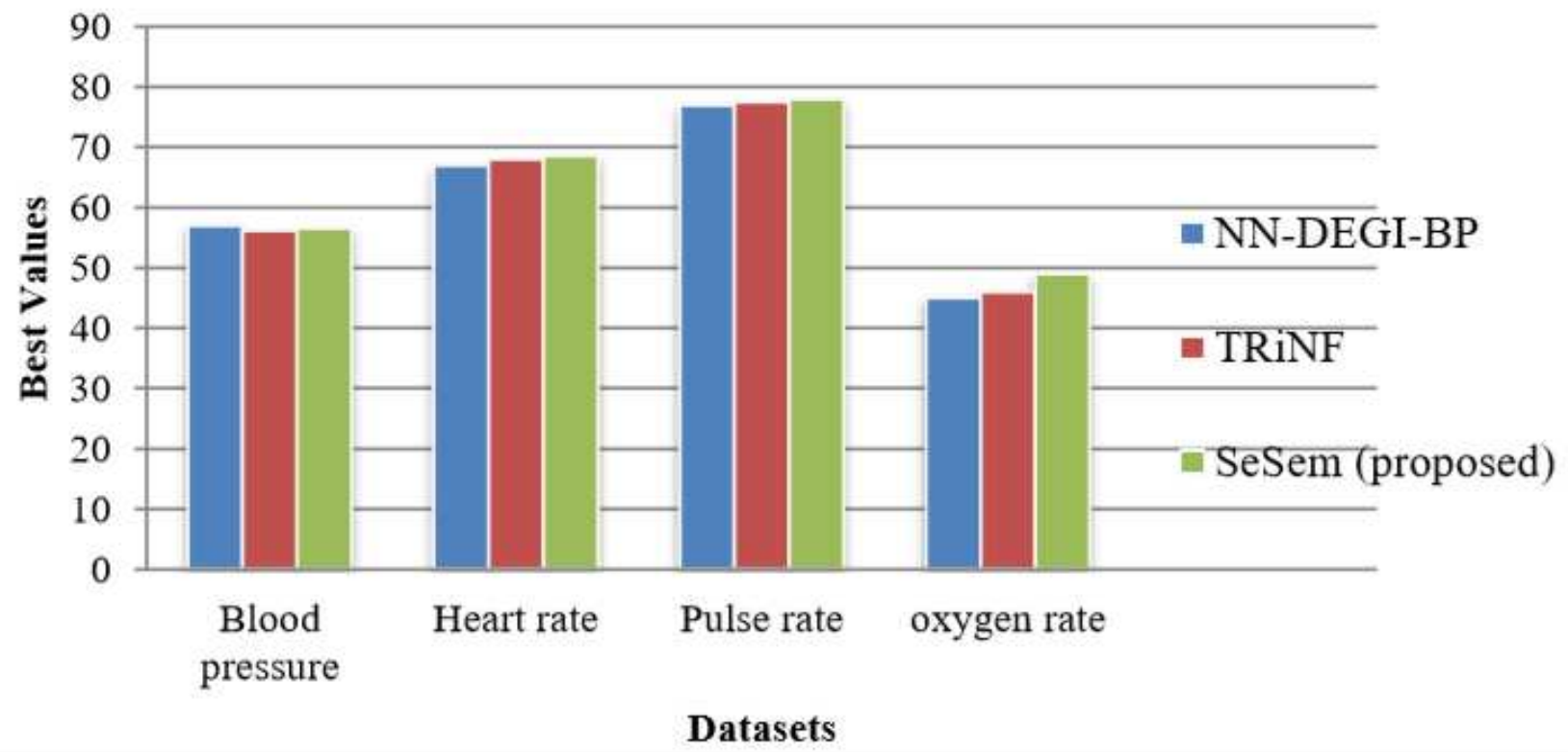

Figure 4

Best PPV Value

Negative Predictive Values (NPV)

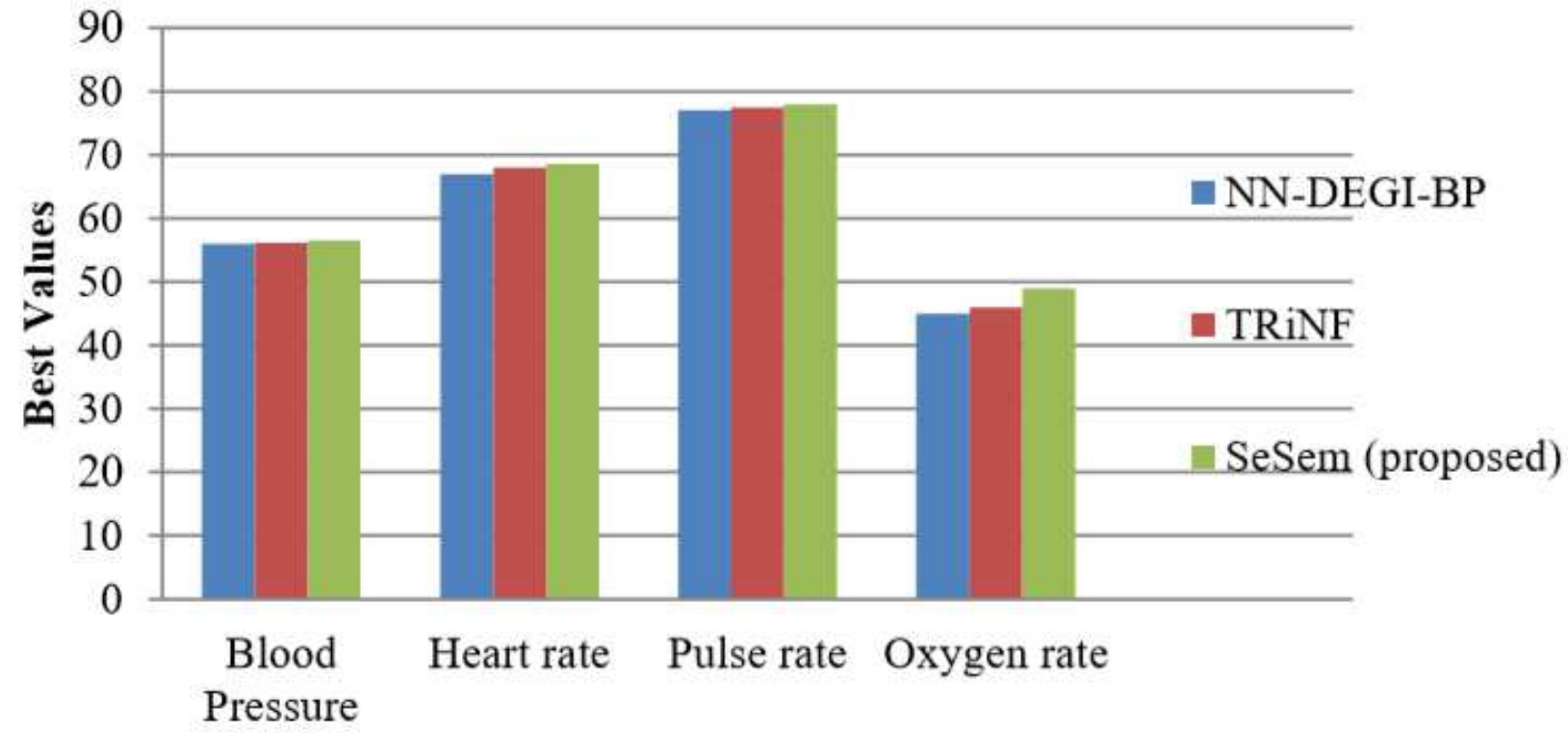

Datasets

Figure 5 


\section{Classifier Accuracy}

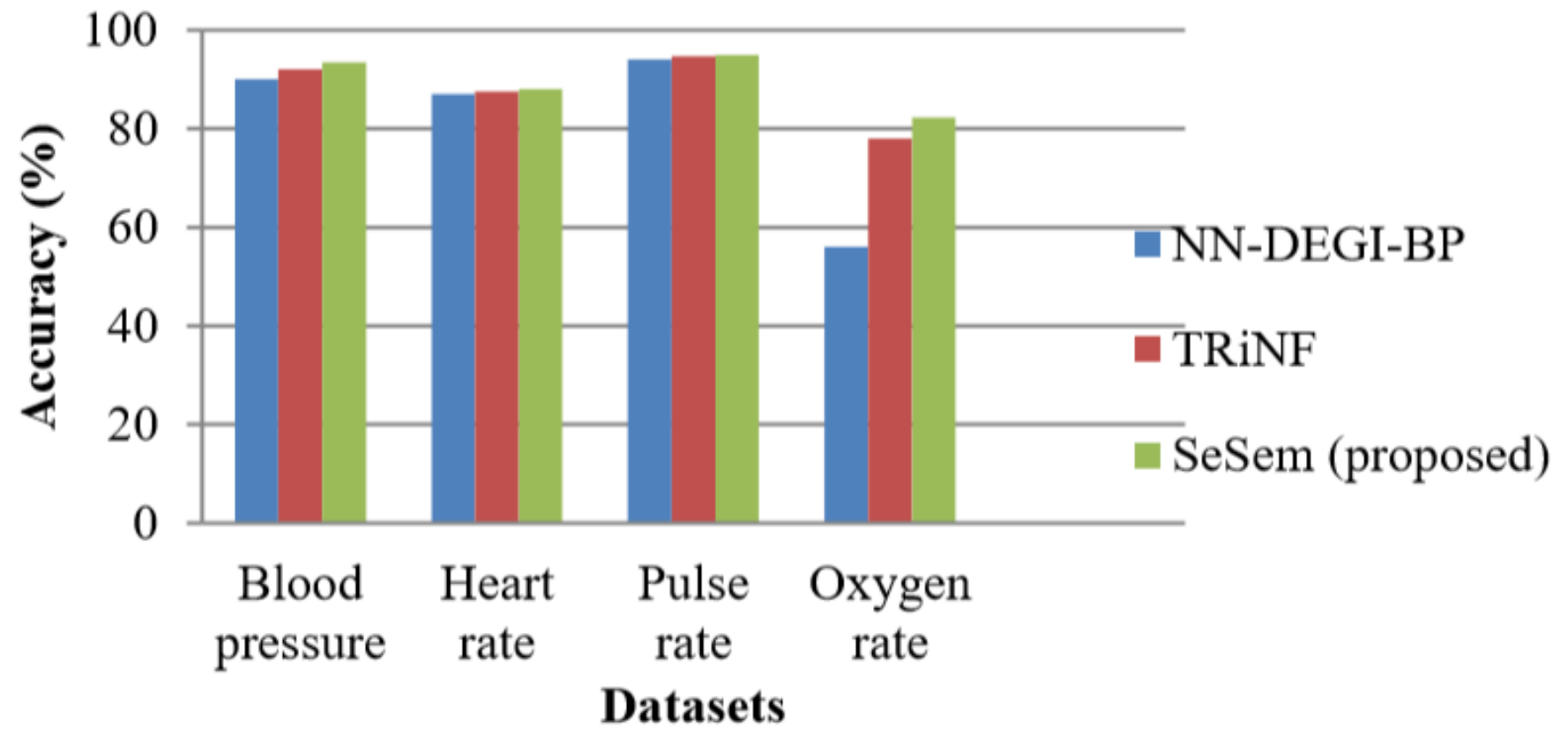

Figure 6

Classifier accuracy for NN-DEGI-BP, TRiNF, and SeSem (proposed) 


\section{Classifier Accuracy}

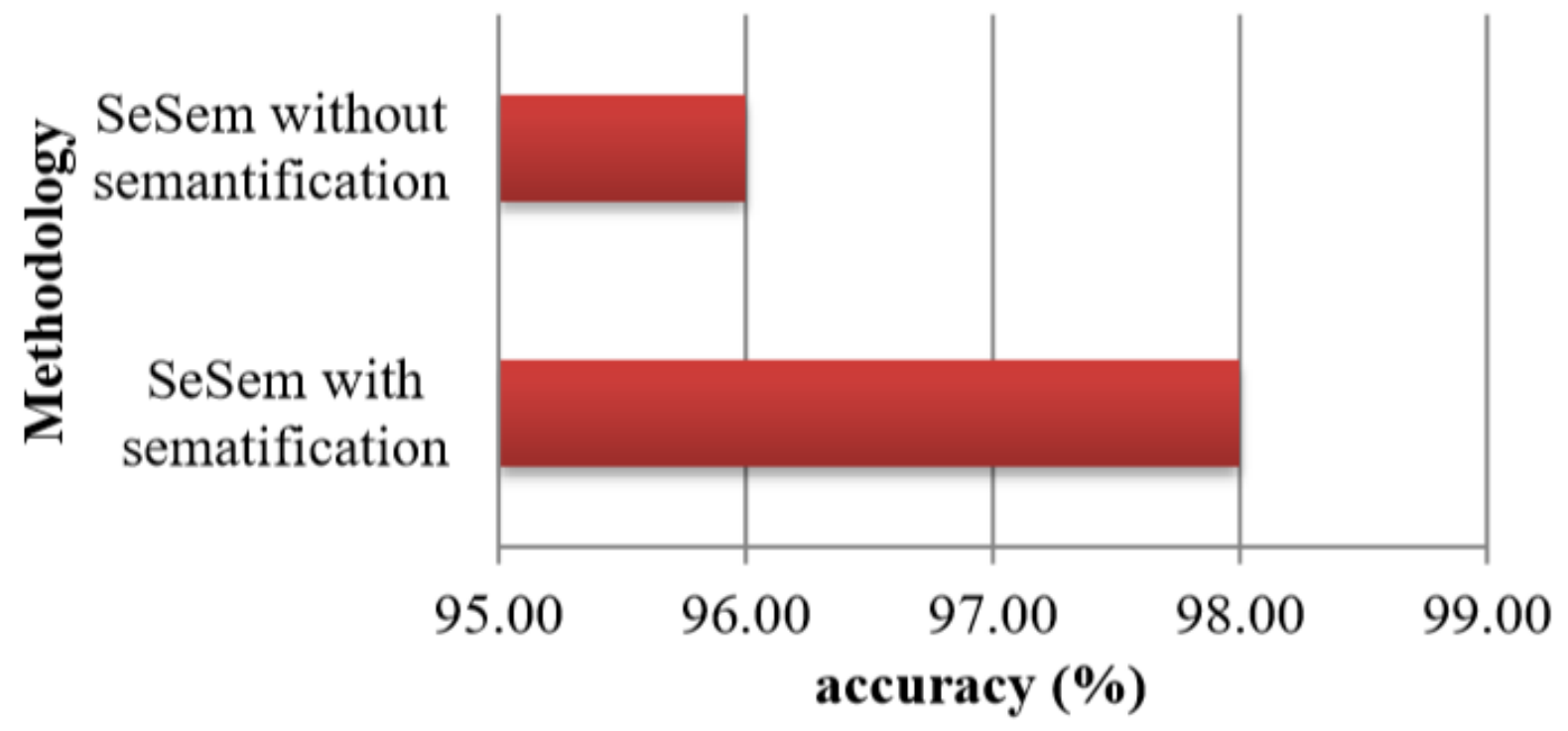

Figure 7

Classifier accuracy with and without semantification

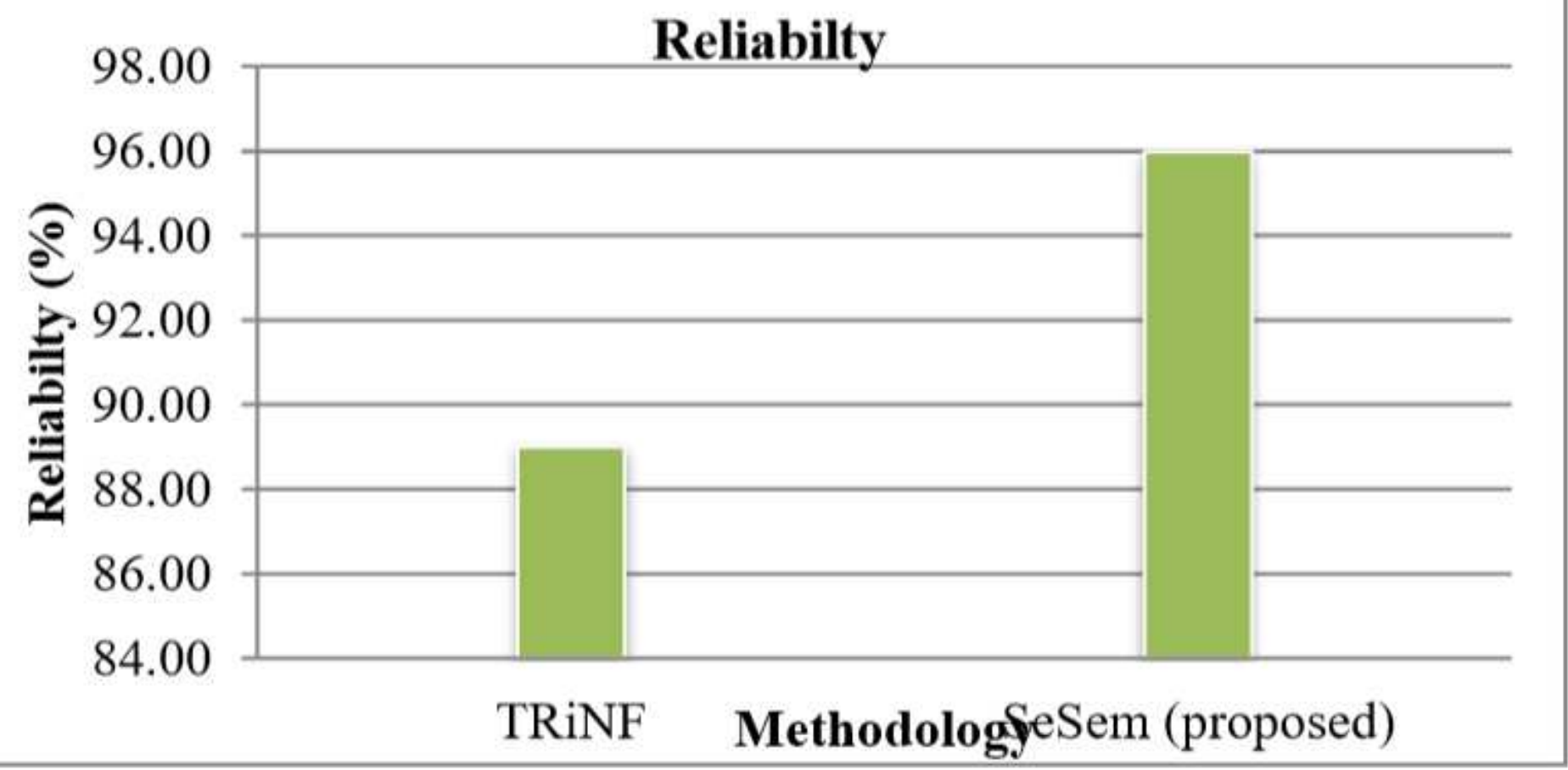

Figure 8 
Reliability

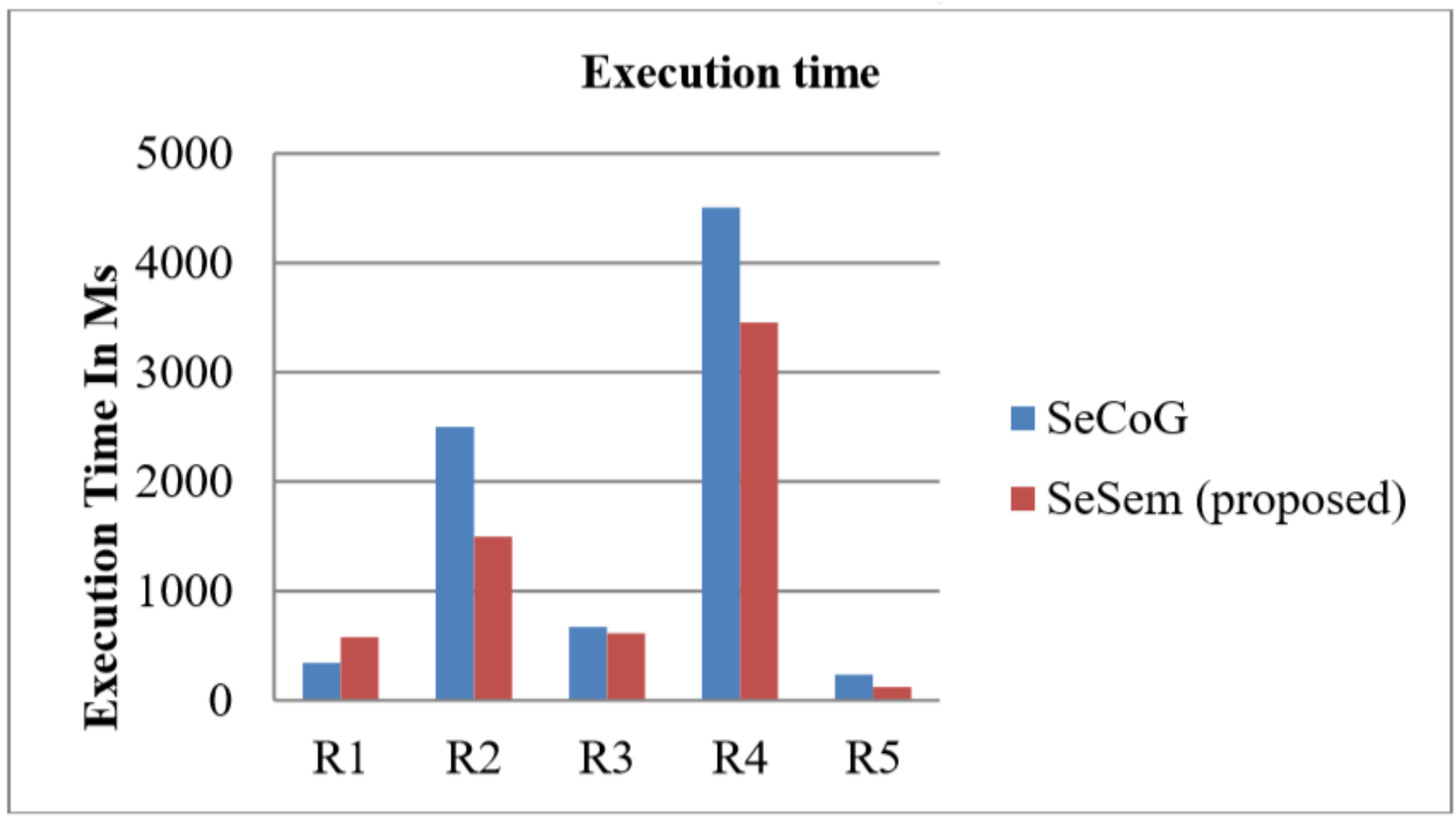

Figure 9

Execution time 


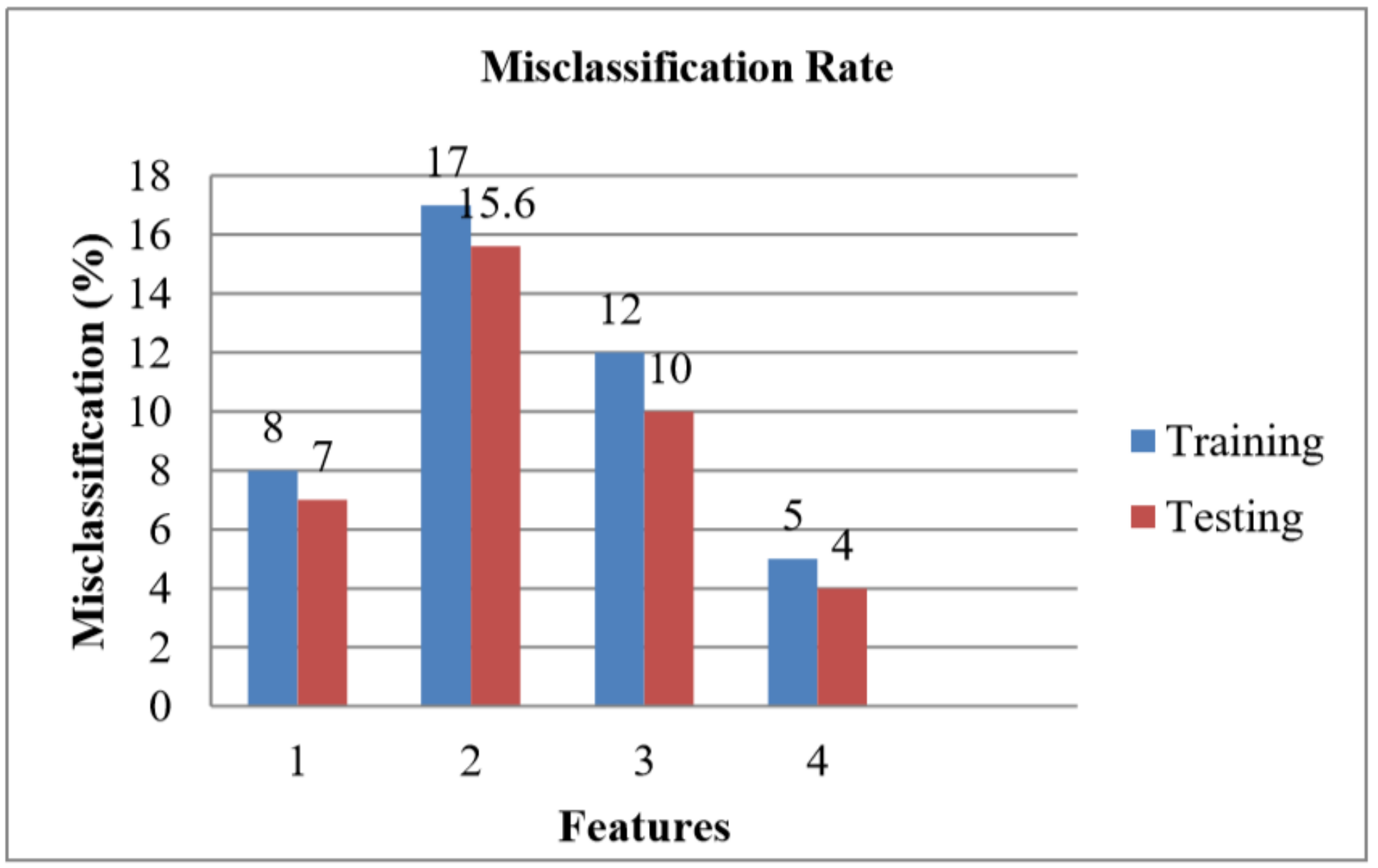

Figure 10

Misclassification Rate 